\title{
GROMOV HYPERBOLICITY THROUGH DECOMPOSITION OF METRIC SPACES II
}

\author{
Ana Portilla, José M. Rodríguez ${ }^{1}$ and Eva Tourís ${ }^{2}$
}

AMS Subj. Classification: 30F20, 30F45

Key Words: Gromov hyperbolicity, metric space, decomposition.

\section{$\S 0$. Abstract}

In this paper we study the hyperbolicity in the Gromov sense of metric spaces. We deduce the hyperbolicity of a space from the hyperbolicity of its "building block components", which can be joined following an arbitrary scheme. These results are especially valuable since they simplify notably the topology and allow to obtain global results from local information. Some interesting theorems about the role of punctures and funnels on the hyperbolicity of Riemann surfaces can be deduced from the conclusions of this paper.

\section{§1. Introduction}

A good way to understand the important connections between graphs and Potential Theory on Riemannian manifolds (see e.g. [ARY], [CFPR], [FR], [HS], [K1], [K2], [S]) is to study the Gromov hyperbolic spaces. This approach allows to establish a general setting to work simultaneously with graphs and manifolds, in the context of metric spaces. Besides, the idea of Gromov hyperbolicity grasps the essence of negatively curved spaces, and has been successfully used in the theory of groups (see e.g. $[\mathrm{GH}]$ and the references therein).

Although there exist some interesting examples of hyperbolic spaces (see the examples after Definition 1), the literature gives no good guide about how to determine whether or not a space is hyperbolic. This limitation can be somehow got round, since the theory allows to obtain powerful results about non-hyperbolic spaces which have hyperbolic universal coverings. As topological "obstacles" may prevent a space from being hyperbolic, the possibility of studying its universal covering instead, which is always free of obstacles, implies a substantial simplification, and sometimes let us extract important information about the space itself (see [P]).

However, as was stated above, the characterization of hyperbolic spaces remains open. Recently, some interesting results about the hyperbolicity of Euclidean bounded domains with their quasihyperbolic metric have made significant progress in this direction (see [BHK] and the references therein).

Originally, we were interested in studying when non-exceptional Riemann surfaces equipped with its Poincaré metric were Gromov hyperbolic. However, we have proved several theorems on hyperbolicity for general metric spaces, which are interesting by themselves and have important consequences for Riemann surfaces (see [PRT]). Although one should expect Gromov hyperbolicity in non-exceptional Riemann surfaces due to its constant curvature -1 , this turns out to be untrue in general, since topological obstacles can impede it: for instance, the two-dimensional jungle-gym (a $\mathbf{Z}^{2}$-covering of a torus with genus two) is not hyperbolic. Let us recall that in the case of modulated plane domains, quasihyperbolic metric and Poincaré metric are equivalent. One can find results on hyperbolicity of Riemann surfaces in [RT] and [PRT].

\footnotetext{
${ }^{1}$ The research of the second author was partially supported by a grant from DGI (BFM 2000-0022) Spain.

${ }^{2}$ The research of the third author was supported by a grant from DGI (BFM 2000-0022) Spain.
} 
Here we present the outline of the main results. We refer to the next sections for the definitions and the precise statements of the theorems.

The main aim in this paper is obtaining global results on hyperbolicity from local information. That was the idea that lead us to think of a space $X$ as the union of some "pieces" or "building block components" $\left\{X_{n}\right\}_{n \in \Lambda}$.

Our first theorem (see Theorem 1) states that if the above mentioned pieces $X_{n}$ are joined together following a tree-like design (that is, avoiding the creation of extra topological obstacles), then the uniform hyperbolicity of the pieces guarantees the hyperbolicity of the global space $X$.

However, if pieces are joined together in a general graph-like style (that is to say, the hypothesis on simple topological connections is removed), the uniform hyperbolicity of pieces is no longer enough to guarantee the hyperbolicity of the global space $X$. But, surprisingly, if $Y$ is a graph that models appropriately the connections among uniformly hyperbolic pieces $X_{n}$ 's, the hyperbolicity of $Y$ let us assure the hyperbolicity of $X$. (This fact turns out to be obvious when $\mathrm{Y}$ is a tree.)

It is noticeable that the graph $Y$ must comply with some metrical requests in order to be an acceptable model for the connections among the pieces $X_{n}$. However, $Y$ is not required at all to model the subspaces themselves (which might be arbitrarily wide far away from the connections). Taking advantage of these facts, Theorem 2 provides a much more general frame, since it does not require that the space $Y$ used as a model to stick the pieces together is a graph.

When applied to Riemann surfaces these theorems let us deduce interesting consequences. In [PRT, Theorems 3.2 and 3.4] we work on the role of punctures and funnels of a Riemann surface in its hyperbolicity. These results allow, in many cases, to forget punctures and funnels in order to analyze the hyperbolicity of a Riemann surface; this fact can be a significant simplification in the topology of the surface, and therefore makes easier the study of its hyperbolicity.

It is a remarkable fact that the constants appearing in the theorems of this paper depend just on a small number of parameters. This is a common place in the theory of hyperbolic spaces (see e.g. theorems A, B and C).

Notations. We denote by $X$ or $X_{n}$ geodesic metric spaces. By $d_{X}, L_{X}$ and $B_{X}$ we shall denote, respectively, the distance, the length and the balls in the metric of $X$.

Finally, we denote by $k_{i}$ positive constants which can assume different values in different theorems.

Acknowledgements. We would like to thank Professors J. L. Fernández and M. V. Melián for some useful discussions.

\section{§2. Results in MetriC SPACES}

In our study of hyperbolic Gromov spaces we use the notations of [GH]. We give now the basic facts about these spaces. We refer to $[\mathrm{GH}]$ for more background and further results.

Definition 1. Let us fix a point $w$ in a metric space $(X, d)$. We define the Gromov product of $x, y \in X$ with respect to the point $w$ as

$$
(x \mid y)_{w}:=\frac{1}{2}(d(x, w)+d(y, w)-d(x, y)) \geq 0 .
$$


We say that the metric space $(X, d)$ is $\delta$-hyperbolic $(\delta \geq 0)$ if

$$
(x \mid z)_{w} \geq \min \left\{(x \mid y)_{w},(y \mid z)_{w}\right\}-\delta
$$

for every $x, y, z, w \in X$. We say that $X$ is hyperbolic (in the Gromov sense) if the value of $\delta$ is not important.

It is convenient to remark that this definition of hyperbolicity is not universally accepted, since sometimes the word hyperbolic refers to negative curvature or to the existence of Green's function. However, in this paper we only use the word hyperbolic in the sense of Definition 1.

Examples: (1) Every bounded metric space $X$ is (diam $X$ )-hyperbolic (see e.g. [GH, p.29]).

(2) Every complete simply connected Riemannian manifold with sectional curvature which is bounded from above by $-k$, with $k>0$, is hyperbolic (see e.g. [GH, p.52]).

(3) Every tree with edges of arbitrary length is 0-hyperbolic (see e.g. [GH, p.29]).

Definition 2. If $\gamma:[a, b] \longrightarrow X$ is a continuous curve in a metric space $(X, d)$, we can define the length of $\gamma$ as

$$
L(\gamma):=\sup \left\{\sum_{i=1}^{n} d\left(\gamma\left(t_{i-1}\right), \gamma\left(t_{i}\right)\right): a=t_{0}<t_{1}<\cdots<t_{n}=b\right\}
$$

We say that $\gamma$ is a geodesic if it is an isometry, i.e. $L\left(\left.\gamma\right|_{[t, s]}\right)=d(\gamma(t), \gamma(s))=|t-s|$ for every $s, t \in[a, b]$. We say that $X$ is a geodesic metric space if for every $x, y \in X$ there exists a geodesic joining $x$ and $y$; we denote by $[x, y]$ any of such geodesics (since we do not require uniqueness of geodesics, this notation is ambiguous, but it is convenient). It is clear that every geodesic metric space is path-connected.

Definition 3. If $X$ is a geodesic metric space and $J=\left\{J_{1}, J_{2}, \ldots, J_{n}\right\}$, with $J_{j} \subseteq X$, we say that $J$ is $\delta$-thin if for every $x \in J_{i}$ we have that $d\left(x, \cup_{j \neq i} J_{j}\right) \leq \delta$. If $x_{1}, x_{2}, x_{3} \in X$, a geodesic triangle $T=\left\{x_{1}, x_{2}, x_{3}\right\}$ is the union of three geodesics $\left[x_{1}, x_{2}\right],\left[x_{2}, x_{3}\right]$ and $\left[x_{3}, x_{1}\right]$. The space $X$ is $\delta$-thin (or satisfies the Rips condition with constant $\delta$ ) if every geodesic triangle in $X$ is $\delta$-thin.

If we have a triangle with two identical vertices, we call it a "bigon". Obviously, every bigon in a $\delta$-thin space is $\delta$-thin.

Definition 4. Given a geodesic triangle $T=\{x, y, z\}$ in a geodesic metric space $X$, let $T_{E}$ be a Euclidean triangle with sides of the same length than $T$. Since there is no possible confusion, we will use the same notation for the corresponding points in $T$ and $T_{E}$. The maximum inscribed circle in $T_{E}$ meets the side $[x, y]$ (respectively $[y, z],[z, x]$ ) in a point $z^{\prime}$ (respectively $x^{\prime}, y^{\prime}$ ) such that $d\left(x, z^{\prime}\right)=d\left(x, y^{\prime}\right), d\left(y, x^{\prime}\right)=d\left(y, z^{\prime}\right)$ and $d\left(z, x^{\prime}\right)=d\left(z, y^{\prime}\right)$. We call the points $x^{\prime}, y^{\prime}, z^{\prime}$, the internal points of $\{x, y, z\}$. There is a unique isometry $f$ of the triangle $\{x, y, z\}$ onto a tripod (a tree with one vertex $w$ of degree 3 , and three vertices $x^{\prime \prime}, y^{\prime \prime}, z^{\prime \prime}$ of degree one, such that $d\left(x^{\prime \prime}, w\right)=d\left(x, z^{\prime}\right)=d\left(x, y^{\prime}\right)$, $d\left(y^{\prime \prime}, w\right)=d\left(y, x^{\prime}\right)=d\left(y, z^{\prime}\right)$ and $\left.d\left(z^{\prime \prime}, w\right)=d\left(z, x^{\prime}\right)=d\left(z, y^{\prime}\right)\right)$. The triangle $\{x, y, z\}$ is $\delta$-fine if $f(p)=f(q)$ implies that $d(p, q) \leq \delta$. The space $X$ is $\delta$-fine if every geodesic triangle in $X$ is $\delta$-fine.

A basic result is that hyperbolicity is equivalent to Rips condition and to be fine: 
Theorem A. ([GH, p.41]) Let us consider a geodesic metric space $X$.

(1) If $X$ is $\delta$-hyperbolic, then it is $4 \delta$-thin and $4 \delta$-fine.

(2) If $X$ is $\delta$-thin, then it is $4 \delta$-hyperbolic and $4 \delta$-fine.

(3) If $X$ is $\delta$-fine, then it is $2 \delta$-hyperbolic and $\delta$-thin.

We present now the class of maps which play the main role in the theory.

Definition 5. A function between two metric spaces $f: X \rightarrow Y$ is a quasi-isometry if there are constants $a \geq 1, b \geq 0$ with

$$
\frac{1}{a} d_{X}\left(x_{1}, x_{2}\right)-b \leq d_{Y}\left(f\left(x_{1}\right), f\left(x_{2}\right)\right) \leq a d_{X}\left(x_{1}, x_{2}\right)+b, \quad \text { for every } x_{1}, x_{2} \in X .
$$

A such function is called an $(a, b)$-quasi-isometry. We say that the image of $f$ is $\varepsilon$-full (for some $\varepsilon \geq 0)$ if for every $y \in Y$ there exists $x \in X$ with $d_{Y}(y, f(x)) \leq \varepsilon$. We say that $X$ and $Y$ are quasi-isometrically equivalents if there exists a quasi-isometry between $X$ and $Y$, with image $\varepsilon$-full, for some $\varepsilon \geq 0$. An $(a, b)$-quasigeodesic in $X$ is an $(a, b)$-quasi-isometry between an interval of $\mathbf{R}$ and $X$. An $(a, b)$-quasigeodesic segment in $X$ is an $(a, b)$-quasi-isometry between a compact interval of $\mathbf{R}$ and $X$.

Let us observe that a quasi-isometry can be discontinuous.

Remark. It is well known (see e.g. [K1], [K2]) that quasi-isometrical equivalence is an equivalence relation. In fact, if $f: X \longrightarrow Y$ is an $(a, b)$-quasi-isometry with image $\varepsilon$-full, then there exists a function $g: Y \longrightarrow X$ which is an $(a, 2 a \varepsilon+a b)$-quasi-isometry. In particular, if $f$ is a surjective $(a, b)$-quasi-isometry, then $g$ is an $(a, a b)$-quasi-isometry (in this case we can choose as $g(y)$ any point in $\left.f^{-1}(y)\right)$.

Quasi-isometries are important since they are the maps which preserve hyperbolicity:

Theorem B. ([GH, p.88]) Let us consider an $(a, b)$-quasi-isometry between two geodesic metric spaces $f: X \longrightarrow Y$. If $Y$ is $\delta$-hyperbolic, then $X$ is $\delta^{\prime}$-hyperbolic, where $\delta^{\prime}$ is a constant which only depends on $\delta, a$ and $b$. Besides, if the image of $f$ is $\varepsilon$-full for some $\varepsilon \geq 0$, then $X$ is hyperbolic if and only if $Y$ is hyperbolic.

It is well-known that if $f$ is not $\varepsilon$-full, the hyperbolicity of $X$ does not imply the hyperbolicity of $Y$ : it is enough to consider the inclusion of $\mathbf{R}$ in $\mathbf{R}^{2}$ (which is indeed an isometry).

Definition 6. Let us consider $H>0$, a metric space $X$, and subsets $Y, Z \subseteq X$. The set $V_{H}(Y):=$ $\{x \in X: d(x, Y) \leq H\}$ is called the H-neighborhood of $Y$ in $X$. The Hausdorff distance of $Y$ to $Z$ is defined by $\mathcal{H}(Y, Z):=\inf \left\{H>0: Y \subseteq V_{H}(Z), Z \subseteq V_{H}(Y)\right\}$.

The following is a beautiful and useful result:

Theorem C. ([GH, p.87]) For each $\delta \geq 0, a \geq 1$ and $b \geq 0$, there exists a constant $H=H(\delta, a, b)$ with the following property:

Let us consider a $\delta$-hyperbolic geodesic metric space $X$ and an $(a, b)$-quasigeodesic $g$ starting in $x$ and finishing in $y$. If $\gamma$ is a geodesic joining $x$ and $y$, then $\mathcal{H}(g, \gamma) \leq H$.

This property is known as geodesic stability. Mario Bonk has proved that, in fact, geodesic stability is equivalent to hyperbolicity [B]. 
Along this paper we will work with topological subspaces of a geodesic metric space $X$. There is a natural way to define a distance in these spaces:

Definition 7. If $X_{0}$ is a path-connected subset of a geodesic metric space $(X, d)$, then we associate to it the restricted distance

$d_{X_{0}}(x, y):=\left.d_{X}\right|_{X_{0}}(x, y):=\inf \left\{L(\gamma): \gamma \subset X_{0}\right.$ is a continuous curve joining $x$ and $\left.y\right\} \geq d_{X}(x, y)$.

Theorem 1 below allows to transfer the study of the hyperbolicity of a certain space $X$ to their "building block components" $X_{n}$ determined by the following definition.

Definition 8. We say that a geodesic metric space $X$ has a decomposition, if there exists a family of geodesic metric spaces $\left\{X_{n}\right\}_{n \in \Lambda}$ with $X=\cup_{n \in \Lambda} X_{n}$ and $X_{n} \cap X_{m}=\cup_{i \in I_{n m}} \eta_{n m}^{i}$, where for each $n \in \Lambda,\left\{\eta_{n m}^{i}\right\}_{m, i}$ are pairwise disjoint closed subsets of $X_{n}$ ( $\eta_{n m}^{i}=\varnothing$ is allowed); furthermore any geodesic segment in $X$ meets at most a finite number of $\eta_{n m}^{i}$ 's.

We say that $X_{n}$, with $n \in \Lambda$, is a $\left(k_{1}, k_{2}, k_{3}\right)$-tree-piece if it satisfies the following properties:

(a) $\sharp I_{n m} \leq 1$ (then we can write $\eta_{n m}^{i}=\eta_{n m}$ ); if $\sharp I_{n m}=1$, then $X \backslash \eta_{n m}$ is not connected and $a, b$ are in different connected components of $X \backslash \eta_{n m}$ for any $a \in X_{n} \backslash \eta_{n m}, b \in X_{m} \backslash \eta_{n m}$.

(b) $\operatorname{diam}_{X_{n}}\left(\eta_{n m}\right) \leq k_{1}$ for every $m \neq n$, and there exists $A_{n} \subseteq \Lambda$, such that $\operatorname{diam}_{X_{n}}\left(\eta_{n m}\right) \leq$ $k_{2} d_{X_{n}}\left(\eta_{n m}, \eta_{n k}\right)$ if $m \neq k$ and $m, k \in A_{n}$, and $\sum_{m \notin A_{n}} \operatorname{diam}_{X_{n}}\left(\eta_{n m}\right) \leq k_{3}$.

We say that a geodesic metric space $X$ has a tree-decomposition if it has a decomposition and there exist positive constants $k_{1}, k_{2}, k_{3}$, such that every $X_{n}$, with $n \in \Lambda$, is a $\left(k_{1}, k_{2}, k_{3}\right)$-tree-piece.

We wish to emphasize that condition $\operatorname{diam}_{X_{n}}\left(\eta_{n m}\right) \leq k_{1}$ is not very restrictive: if the space is "wide" at every point (in the sense of long injectivity radius, as in the case of simply connected spaces) or "narrow" at every point (as in the case of trees), it is easier to study its hyperbolicity; if we can found narrow parts (as $\eta_{n m}$ ) and wide parts, the problem is more difficult and interesting.

\section{Remarks.}

1. Obviously, condition $(b)$ is required only for $\eta_{n m}, \eta_{n k} \neq \varnothing$.

2. The sets $\Lambda$ and $A_{n}$ do not need to be countable.

3. The hypothesis $\operatorname{diam}_{X_{n}}\left(\eta_{n m}\right) \leq k_{2} d_{X_{n}}\left(\eta_{n m}, \eta_{n k}\right)$ holds if we have $d_{X_{n}}\left(\eta_{n m}, \eta_{n k}\right) \geq k_{2}^{\prime}$, since $\operatorname{diam}_{X_{n}}\left(\eta_{n m}\right) \leq k_{1}$.

4. Condition ( $a$ ) for every $n \in \Lambda$ guarantees that the graph $R=(V, E)$ constructed in the following way is a tree: $V=\cup_{n \in \Lambda}\left\{v_{n}\right\}$ and $\left[v_{n}, v_{m}\right] \in E$ if and only if $\eta_{n m} \neq \varnothing$.

The following result is an improvement of Theorem 2.4 in [RT], using a completely different line of argument; furthermore, this proof provides an explicit expression for the constants involved. It will be used in the proof of Theorem 2 and can be applied to the study of the hyperbolicity of Riemann surfaces (see [PRT, Propositions 3.1 and 3.2]).

Theorem 1. Let us consider a geodesic metric space $X$ with a tree-decomposition $\left\{X_{n}\right\}_{n \in \Lambda}$. Then $X$ is $\delta$-hyperbolic if and only if there exists a constant $k_{4}$ such that $X_{n}$ is $k_{4}$-hyperbolic for every $n \in \Lambda$. 
Furthermore, if $X_{n}$ is $k_{4}$-hyperbolic, we can take $\delta=4\left(2 k_{1}+4 k_{4}+2 H\left(k_{4}, 2 \max \left\{1, k_{2}\right\}, 4 k_{1}+2 k_{3}\right)\right)$, where $H$ is the constant in Theorem $C$; if $X$ is $\delta$-hyperbolic, we can take $k_{4}=16 \max \left\{1, k_{2}\right\}\left(2 \delta+k_{1}+\right.$ $\left.k_{3}+H\left(\delta, 2 \max \left\{1, k_{2}\right\}, 2 k_{1}+2 k_{3}\right)\right)$.

Observe that the sets $\eta_{n m}$ do not need to be connected and therefore we can create a finite number of "handles" each time we paste two pieces.

The conclusion of Theorem 1 is not true without hypothesis $(b)$ in Definition 8 , as it is shown in following examples:

The set $Q=\{z=x+i y: x \geq 0, y \geq 0\}$, with its Euclidean distance, is not hyperbolic, but $Q$ is the union of the 1-thin pieces $X_{n}=\{z \in Q: n-1 \leq|z| \leq n\}$.

Let us consider any funnel $F$ with boundary $\gamma$. The results on [RT] gives that $F$ is hyperbolic. However, it is the union of the non-uniformly hyperbolic pieces $X_{n}=\{z \in F: n-1 \leq d(z, \gamma) \leq n\}$ (the hyperbolicity constant of $X_{n}$ is comparable with $L\left(\partial X_{n}\right)$ ).

The proof of Theorem 1 gives the following results.

Corollary 1. Let us consider a geodesic metric space $X$ with a decomposition $\left\{X_{n}\right\}_{n \in \Lambda}$. Let us assume that, for some fixed $n_{0} \in \Lambda, X_{n_{0}}$ is a $\left(k_{1}, k_{2}, k_{3}\right)$-tree-piece and it is $k_{4}$-hyperbolic. If $T$ is a geodesic triangle in $X$ and $X_{n_{0}}$ intersects at least two sides of $T$, then $X_{n_{0}} \cap T$ is $\delta^{*}$-thin, with $\delta^{*}:=3 k_{1} / 2+4 k_{4}+2 H\left(k_{4}, 2 \max \left\{1, k_{2}\right\}, 4 k_{1}+2 k_{3}\right)$.

Corollary 2. Let us consider a $\delta$-hyperbolic geodesic metric space $X$ with a decomposition $\left\{X_{n}\right\}_{n \in \Lambda}$. Let us assume that, for some fixed $n_{0} \in \Lambda, X_{n_{0}}$ is a $\left(k_{1}, k_{2}, k_{3}\right)$-tree-piece. Then $X_{n_{0}}$ is $\delta_{0}$-thin, with $\delta_{0}:=4 \max \left\{1, k_{2}\right\}\left(2 \delta+k_{1}+k_{3}+H\left(\delta, 2 \max \left\{1, k_{2}\right\}, 2 k_{1}+2 k_{3}\right)\right)$.

In order to prove Theorem 1 we need some technical results.

Lemma 1. Let us consider a geodesic metric space $X$ and a geodesic $\eta=\left[x_{0}, x_{2 n+1}\right]=\cup_{j=1}^{2 n+1}\left[x_{j-1}, x_{j}\right]$ For each $1 \leq j \leq n$, let us consider a continuous curve $\eta_{j}^{\prime}$ joining $x_{2 j-1}$ and $x_{2 j}$, such that $L\left(\eta_{j}^{\prime}\right) \leq a$ for every $1 \leq j \leq n$ and $L\left(\eta_{j}^{\prime}\right) \leq b L\left(\left[x_{2 j}, x_{2 j+1}\right]\right)$ for every $1 \leq j \leq n-1$. If $\eta^{\prime}$ is the curve obtained from $\eta$ by replacing $\left[x_{2 j-1}, x_{2 j}\right]$ by $\eta_{j}^{\prime}$, then $\eta^{\prime}$ is a continuous $(2 \max \{1, b\}, 2 a)$-quasigeodesic with its arc-length parametrization.

Proof of Lemma 1. Let us consider the arc-length parametrizations $\eta:[0, l] \longrightarrow X$ and $\eta^{\prime}$ : $\left[0, l^{\prime}\right] \longrightarrow X$. We can write $[0, l]=\cup_{j=1}^{2 n+1}\left[t_{j-1}, t_{j}\right]$ and $\left[0, l^{\prime}\right]=\cup_{j=1}^{2 n+1}\left[l_{j-1}, l_{j}\right]$, such that $\eta^{\prime}\left(l_{j}\right)=\eta\left(t_{j}\right)=$ $x_{j}$ for every $0 \leq j \leq 2 n+1, \eta^{\prime}\left(\left[l_{2 j}, l_{2 j+1}\right]\right)=\left[x_{2 j}, x_{2 j+1}\right]$ for every $0 \leq j \leq n$ and $\eta^{\prime}\left(\left[l_{2 j-1}, l_{2 j}\right]\right)=\eta_{j}^{\prime}$ for every $1 \leq j \leq n$. The hypothesis give that $l_{2 j}-l_{2 j-1} \leq a$ for every $1 \leq j \leq n$ and $l_{2 j}-l_{2 j-1} \leq$ $b\left(l_{2 j+1}-l_{2 j}\right)$ for every $1 \leq j \leq n-1$.

Since we consider $\eta^{\prime}$ with its arc-length parametrization, then, for every $s, t \in\left[0, l^{\prime}\right]$, we have $d\left(\eta^{\prime}(t), \eta^{\prime}(s)\right) \leq L\left(\eta^{\prime}([s, t])\right)=|t-s|$.

If $s, t \in\left[l_{2 j}, l_{2 j+1}\right]$, then $d\left(\eta^{\prime}(t), \eta^{\prime}(s)\right)=|t-s|$ for every $0 \leq j \leq n$.

If $s \in\left[l_{2 i}, l_{2 i+1}\right]$ and $t \in\left[l_{2 j}, l_{2 j+1}\right]$, without loss of generality, we can assume that $i<j$; then there 
exist $s^{\prime} \in\left[t_{2 i}, t_{2 i+1}\right]$ and $t^{\prime} \in\left[t_{2 j}, t_{2 j+1}\right]$ such that

$$
\begin{aligned}
d\left(\eta^{\prime}(t), \eta^{\prime}(s)\right)= & d\left(\eta\left(t^{\prime}\right), \eta\left(s^{\prime}\right)\right) \geq t_{2 i+1}-s^{\prime}+\sum_{k=i+1}^{j-1}\left(t_{2 k+1}-t_{2 k}\right)+t^{\prime}-t_{2 j} \\
= & l_{2 i+1}-s+\frac{1}{2} \sum_{k=i+1}^{j-1}\left(l_{2 k+1}-l_{2 k}+l_{2 k+1}-l_{2 k}\right)+t-l_{2 j} \\
\geq & \frac{1}{2}\left(l_{2 i+1}-s+b^{-1} \sum_{k=i+1}^{j-1}\left(l_{2 k}-l_{2 k-1}\right)+\sum_{k=i+1}^{j-1}\left(l_{2 k+1}-l_{2 k}\right)\right. \\
& \left.+l_{2 j}-l_{2 j-1}-a+t-l_{2 j}\right) \geq \frac{1}{2} \min \left\{1, b^{-1}\right\}(t-s)-\frac{a}{2} .
\end{aligned}
$$

In the general case, if $s, t \in\left[0, l^{\prime}\right]$ there exist $s^{*} \in\left[l_{2 i}, l_{2 i+1}\right]$ and $t^{*} \in\left[l_{2 j}, l_{2 j+1}\right]$, with $\left|s-s^{*}\right| \leq a / 2$ and $\left|t-t^{*}\right| \leq a / 2$. Hence

$$
\begin{aligned}
d\left(\eta^{\prime}(t), \eta^{\prime}(s)\right) & \geq d\left(\eta^{\prime}\left(t^{*}\right), \eta^{\prime}\left(s^{*}\right)\right)-a \geq \frac{1}{2} \min \left\{1, b^{-1}\right\}\left|t^{*}-s^{*}\right|-\frac{3 a}{2} \\
& \geq \frac{1}{2} \min \left\{1, b^{-1}\right\}|t-s|-2 a .
\end{aligned}
$$

Lemma 2. Let us consider a geodesic metric space $X$ and a continuous $(a, b)$-quasigeodesic with its arc-length parametrization $\eta:[0, l] \longrightarrow X$, such that $[0, l]=\cup_{j=1}^{2 n+1}\left[t_{j-1}, t_{j}\right]$. For each $1 \leq j \leq n$, let us consider a continuous curve $\eta_{j}^{\prime}$ joining $\eta\left(t_{2 j-1}\right)$ and $\eta\left(t_{2 j}\right)$ such that $\sum_{j=1}^{n} L\left(\eta_{j}^{\prime}\right) \leq c$. If $\eta^{\prime}$ is the curve obtained from $\eta$ by substituting $\eta\left(\left[t_{2 j-1}, t_{2 j}\right]\right)$ by $\eta_{j}^{\prime}$, then $\eta^{\prime}$ is a continuous $\left(a, b+\left(1+3 a^{-1}\right) c / 2\right)$ quasigeodesic with its arc-length parametrization.

Proof of Lemma 2. Let us consider the arc-length parametrization $\eta^{\prime}:\left[0, l^{\prime}\right] \longrightarrow X$. We can write $\left[0, l^{\prime}\right]=\cup_{j=1}^{2 n+1}\left[l_{j-1}, l_{j}\right]$, such that $\eta^{\prime}\left(l_{j}\right)=\eta\left(t_{j}\right)$ for every $0 \leq j \leq 2 n+1, \eta^{\prime}\left(\left[l_{2 j}, l_{2 j+1}\right]\right)=\eta\left(\left[t_{2 j}, t_{2 j+1}\right]\right)$ for every $0 \leq j \leq n$ and $\eta^{\prime}\left(\left[l_{2 j-1}, l_{2 j}\right]\right)=\eta_{j}^{\prime}$ for every $1 \leq j \leq n$. We have that $\sum_{j=1}^{n}\left(l_{2 j}-l_{2 j-1}\right) \leq c$.

Since we consider $\eta^{\prime}$ with its arc-length parametrization, then, for every $s, t \in\left[0, l^{\prime}\right]$, we have that $d\left(\eta^{\prime}(t), \eta^{\prime}(s)\right) \leq L\left(\eta^{\prime}([s, t])\right)=|t-s|$. In order to prove the other inequality, we have:

If $s, t \in\left[l_{2 j}, l_{2 j+1}\right]$, then $d\left(\eta^{\prime}(t), \eta^{\prime}(s)\right) \geq a^{-1}|t-s|-b$ for every $0 \leq j \leq n$.

If $s \in\left[l_{2 i}, l_{2 i+1}\right]$ and $t \in\left[l_{2 j}, l_{2 j+1}\right]$, without loss of generality we can assume that $i<j$; then there exist $s^{\prime} \in\left[t_{2 i}, t_{2 i+1}\right]$ and $t^{\prime} \in\left[t_{2 j}, t_{2 j+1}\right]$ such that

$$
\begin{aligned}
d\left(\eta^{\prime}(t), \eta^{\prime}(s)\right) & =d\left(\eta\left(t^{\prime}\right), \eta\left(s^{\prime}\right)\right) \geq a^{-1}\left|t^{\prime}-s^{\prime}\right|-b \\
& =a^{-1}\left(t^{\prime}-t_{2 j}+\sum_{k=2 i+1}^{2 j-1}\left(t_{k+1}-t_{k}\right)+t_{2 i+1}-s^{\prime}\right)-b \\
& \geq a^{-1}\left(t-l_{2 j}+\sum_{k=2 i+1}^{2 j-1}\left(l_{k+1}-l_{k}\right)+l_{2 i+1}-s\right)-\left(b+a^{-1} c\right) \\
& =a^{-1}(t-s)-\left(b+a^{-1} c\right) .
\end{aligned}
$$

In the general case, if $s, t \in\left[0, l^{\prime}\right]$, there exist $s^{*} \in\left[l_{2 i}, l_{2 i+1}\right]$ and $t^{*} \in\left[l_{2 j}, l_{2 j+1}\right]$, with $\left|s-s^{*}\right|+\mid t-$ $t^{*} \mid \leq c / 2$. Hence

$$
\begin{aligned}
d\left(\eta^{\prime}(t), \eta^{\prime}(s)\right) & \geq d\left(\eta^{\prime}\left(t^{*}\right), \eta^{\prime}\left(s^{*}\right)\right)-c / 2 \geq a^{-1}\left|t^{*}-s^{*}\right|-\left(b+a^{-1} c+c / 2\right) \\
& \geq a^{-1}|t-s|-\left(b+3 a^{-1} c / 2+c / 2\right) .
\end{aligned}
$$


Lemma 3. Let us consider an $(a, b)$-quasigeodesic $q_{1}:[\alpha, \beta] \longrightarrow X$ and two continuous curves with arc-length parametrization $q_{0}:\left[\alpha-d_{1}, \alpha\right] \longrightarrow X, q_{2}:\left[\beta, \beta+d_{2}\right] \longrightarrow X$, verifying $q_{0}(\alpha)=q_{1}(\alpha)$ and $q_{2}(\beta)=q_{1}(\beta)$. Then the curve $q:=q_{0} \cup q_{1} \cup q_{2}$ is an $\left(a, b+\left(1+a^{-1}\right)\left(d_{1}+d_{2}\right)\right)$-quasigeodesic.

Proof of Lemma 3. We consider the case $s \in\left[\alpha-d_{1}, \alpha\right]$ and $t \in\left[\beta, \beta+d_{2}\right]$, since the other cases are easier.

$$
\begin{aligned}
d(q(t), q(s)) & \leq d\left(q(t), q_{1}(\beta)\right)+d\left(q_{1}(\beta), q_{1}(\alpha)\right)+d\left(q_{1}(\alpha), q(s)\right) \\
& \leq d_{2}+a(\beta-\alpha)+b+d_{1} \leq a(t-s)+b+d_{1}+d_{2} \\
d(q(t), q(s)) & \geq d\left(q_{1}(\beta), q_{1}(\alpha)\right)-d\left(q(t), q_{1}(\beta)\right)-d\left(q_{1}(\alpha), q(s)\right) \\
& \geq a^{-1}(\beta-\alpha)-b-d_{1}-d_{2} \geq a^{-1}(t-s)-a^{-1}\left(d_{1}+d_{2}\right)-b-d_{1}-d_{2} .
\end{aligned}
$$

Definition 9. Let us consider three quasigeodesic segments $J_{1}$ joining $x_{1}$ and $x_{2}^{\prime}, J_{2}$ joining $x_{2}$ and $x_{3}^{\prime}, J_{3}$ joining $x_{3}$ and $x_{1}^{\prime}$, in a metric space. We say that $T=\left\{J_{1}, J_{2}, J_{3}\right\}$ is an $(a, b, c)$-quasigeodesic triangle if $J_{1}, J_{2}, J_{3}$ are $(a, b)$-quasigeodesics and $d\left(x_{i}, x_{i}^{\prime}\right) \leq c$ for $1 \leq i \leq 3$.

Lemma 4. For each $\delta, b, c \geq 0$ and $a \geq 1$, there exists a constant $K=K(\delta, a, b, c)$ with the following property:

If $X$ is a $\delta$-hyperbolic geodesic metric space and $T \subseteq X$ is an $(a, b, c)$-quasigeodesic triangle, then $T$ is $K$-thin. Furthermore, $K=4 \delta+c+2 H(\delta, a, b+2 c)$, where $H$ is the constant in Theorem $C$.

Proof of Lemma 4. We consider three geodesic segments $\left[x_{2}^{\prime}, x_{2}\right],\left[x_{3}^{\prime}, x_{3}\right]$ and $\left[x_{1}^{\prime}, x_{1}\right]$. By Lemma 3 (with $d_{1}=0$ and $d_{2} \leq c$ ), the curves $s\left(x_{1}, x_{2}\right):=J_{1} \cup\left[x_{2}^{\prime}, x_{2}\right], s\left(x_{2}, x_{3}\right):=J_{2} \cup\left[x_{3}^{\prime}, x_{3}\right]$ and $s\left(x_{3}, x_{1}\right):=J_{3} \cup\left[x_{1}^{\prime}, x_{1}\right]$ are $(a, b+2 c)$-quasigeodesics. By Theorem $\mathrm{C}$, there exist geodesics $\left\{\left[x_{1}, x_{2}\right],\left[x_{2}, x_{3}\right],\left[x_{3}, x_{1}\right]\right\}$ with $\mathcal{H}\left(s\left(x_{i}, x_{j}\right),\left[x_{i}, x_{j}\right]\right) \leq H$, for some constant $H=H(\delta, a, b+2 c)$.

We prove now that the $(a, b+2 c, 0)$-quasigeodesic triangle $T^{\prime}=\left\{J_{1}, J_{2}, J_{3}\right\}$ is $(4 \delta+2 H)$-thin. Let us consider any permutation $\left\{x_{i}, x_{j}, x_{k}\right\}$ of $\left\{x_{1}, x_{2}, x_{3}\right\}$ and $x \in s\left(x_{i}, x_{j}\right)$; then there exists $x^{\prime} \in\left[x_{i}, x_{j}\right]$ with $d\left(x, x^{\prime}\right) \leq H$.

Since the geodesics $\left\{\left[x_{1}, x_{2}\right],\left[x_{2}, x_{3}\right],\left[x_{3}, x_{1}\right]\right\}$ are a geodesic triangle $4 \delta$-thin, there exists $y^{\prime} \in$ $\left[x_{j}, x_{k}\right] \cup\left[x_{k}, x_{i}\right]$ with $d\left(x^{\prime}, y^{\prime}\right) \leq 4 \delta$. Now we can choose $y \in s\left(x_{j}, x_{k}\right) \cup s\left(x_{k}, x_{i}\right)$ with $d\left(y, y^{\prime}\right) \leq H$. Hence, $T^{\prime}$ is $(4 \delta+2 H)$-thin.

Consequently, $T$ is $K$-thin, with $K:=4 \delta+c+2 H$, since $\left[x_{2}^{\prime}, x_{2}\right],\left[x_{3}^{\prime}, x_{3}\right]$ and $\left[x_{1}^{\prime}, x_{1}\right]$ have length less or equal than $c$.

Definition 10. Let us assume that we have a triangle $T$ (not necessarily geodesic) with vertices $\left\{x_{1}, x_{2}, x_{3}\right\}$; we denote by $x_{i} x_{j}$ the side of $T$ joining $x_{i}$ with $x_{j}$. We consider now another triangle $T^{\prime}$ with vertices $\left\{x_{1}^{\prime}, x_{2}^{\prime}, x_{3}^{\prime}\right\}$ such that $x_{i}^{\prime} x_{j}^{\prime}$ is obtained by a certain kind of modification of $x_{i} x_{j}$. We say that $z \in T$ and $z^{\prime} \in T^{\prime}$ are in corresponding sides if $z \in x_{i} x_{j}$ and $z^{\prime} \in x_{i}^{\prime} x_{j}^{\prime}$ for some $i, j$.

Proof of Theorem 1. Let us assume that $X_{n}$ is $k_{4}$-hyperbolic for every $n \in \Lambda$.

We consider a geodesic triangle $T=\{a, b, c\}$ in $X$. We fix $z \in T$; if $z$ belongs to two sides of $T$, there is nothing to prove; if $z$ only belongs to one side of $T$, we denote by $A$ the union of the sides of $T$ which does not intersect $z$. Without loss of generality we can assume that $z \in[a, b]$.

If $T \subseteq X_{n}$ for some $n$, then $T$ is $4 k_{4}$-thin, by Theorem A. 
We assume that $T$ intersects several $X_{n}$ 's. We intend to study $T$ in each of those $X_{n}$ 's separately.

Let us fix $n \in \Lambda$. We consider first the case in which every side of $T$ intersect $X_{n}$. We construct a quasigeodesic triangle $T_{n} \subseteq X_{n}$ modifying $T$ in the following way: If $[a, b] \subseteq X_{n}$, we consider $\left[a_{n}, b_{n}\right]=[a, b]$. If $[a, b]$ is not contained in $X_{n}$, then we consider $g:[0, l] \longrightarrow X$ as an oriented geodesic joining $a$ and $b$. By hypothesis, the geodesic segment $g$ meets at most a finite number of $\eta_{n m}$ 's. Let us define

$$
t_{0}:=\min \left\{0 \leq t \leq l: g(t) \in X_{n}\right\}, \quad t_{l}:=\max \left\{0 \leq t \leq l: g(t) \in X_{n}\right\} .
$$

First of all, let us assume that $g$ meets $\cup_{m \in A_{n}} \eta_{n m}$. We define

$$
t_{1}^{1}:=\min \left\{t_{0} \leq t \leq t_{l}: g(t) \in \cup_{m \in A_{n}} \eta_{n m}\right\} .
$$

There exists this minimum since $g$ is a continuous function in a compact interval and $g \cap\left(\cup_{m \in A_{n}} \eta_{n m}\right)$ is a compact set: each $\eta_{n m}$ is a closed set and $g$ meets at most a finite number of $\eta_{n m}$ 's.

Then there is $m_{1} \in A_{n}$ such that $g\left(t_{1}^{1}\right) \in \eta_{n m_{1}}$, and we define

$$
t_{1}^{2}:=\max \left\{t_{0} \leq t \leq t_{l}: g(t) \in \eta_{n m_{1}}\right\}
$$

In a similar way, we define recursively

$$
t_{i}^{1}:=\min \left\{t_{i-1}^{2}<t \leq t_{l}: g(t) \in \cup_{m \in A_{n}} \eta_{n m}\right\}
$$

if $g\left(t_{i}^{1}\right) \in \eta_{n m_{i}}$, for some $m_{i} \in A_{n}$, we take

$$
t_{i}^{2}:=\max \left\{t_{i-1}^{2}<t \leq t_{l}: g(t) \in \eta_{n m_{i}}\right\} .
$$

We can continue this choice for $1 \leq i \leq r$. We define $g^{\prime}$ as the restriction of $g$ to the closed set $\left[t_{0}, t_{1}^{1}\right] \cup\left[t_{1}^{2}, t_{2}^{1}\right] \cup \cdots \cup\left[t_{r-1}^{2}, t_{r}^{1}\right] \cup\left[t_{r}^{2}, t_{l}\right]$. Observe that $g^{\prime} \subseteq X_{n}$. Now, let us choose geodesics $g_{i}$ in $X_{n}$ connecting $g\left(t_{i}^{1}\right)$ and $g\left(t_{i}^{2}\right)$. We define $\gamma:=g^{\prime} \cup g_{1} \cup g_{2} \cup \cdots \cup g_{r}$. By Lemma 1, we have that $\gamma:[0, L] \longrightarrow X_{n}$ is a continuous $\left(2 \max \left\{1, k_{2}\right\}, 2 k_{1}\right)$-quasigeodesic with its arc-length parametrization (observe that $\gamma(0)=g\left(t_{0}\right)$ and $\left.\gamma(L)=g\left(t_{l}\right)\right)$.

If $g$ does not meet $\cup_{m \in A_{n}} \eta_{n m}$ (or if $t_{i}^{1}=t_{i}^{2}$ for $1 \leq i \leq r$ ), we take $\gamma=g$.

We assume now that $\gamma$ meets $\cup_{m \notin A_{n}} \eta_{n m}$. If we repeat the previous argument, then we can find a $m^{1} \notin A_{n}$ for which we have

$$
s_{1}^{1}:=\min \left\{0 \leq s \leq L: \gamma(s) \in \eta_{n m^{1}}\right\}, \quad s_{1}^{2}:=\max \left\{0 \leq s \leq L: \gamma(s) \in \eta_{n m^{1}}\right\} .
$$

In a similar way, there exist $m^{2}, \ldots, m^{j}$ for which we define recursively for $i=2, \ldots, j$,

$$
s_{i}^{1}:=\min \left\{s_{i-1}^{2}<s \leq L: \eta(s) \in \eta_{n m^{i}}\right\}, \quad s_{i}^{2}:=\max \left\{s_{i-1}^{2}<s \leq L: \eta(s) \in \eta_{n m^{i}}\right\} .
$$

We define $\gamma^{\prime}$ as a restriction of $\gamma$ to the closed set $\left[0, s_{1}^{1}\right] \cup\left[s_{1}^{2}, s_{2}^{1}\right] \cup \cdots \cup\left[s_{j}^{2}, L\right]$; we also have $\gamma^{\prime} \subseteq X_{n}$. Now, let us choose geodesics $h_{i}$ in $X_{n}$ connecting $\gamma\left(s_{i}^{1}\right)$ and $\gamma\left(s_{i}^{2}\right)$. We define $\alpha_{1}:=\gamma^{\prime} \cup h_{1} \cup h_{2} \cup \cdots \cup h_{j}$. 
If $\alpha_{1}:\left[0, l_{1}\right] \longrightarrow X_{n}$ is its arc-length parametrization, Lemma 2 gives that $\alpha_{1}$ is a $\left(2 \max \left\{1, k_{2}\right\}, 2 k_{1}+\right.$ $2 k_{3}$ )-quasigeodesic.

If $\gamma$ does not meet $\cup_{m \notin A_{n}} \eta_{n m}$ (or if $s_{i}^{1}=s_{i}^{2}$ for $1 \leq i \leq j$ ), we take $\alpha_{1}=\gamma$.

In a similar way, we construct the quasigeodesics $\alpha_{2}:\left[0, l_{2}\right] \longrightarrow X_{n}$ and $\alpha_{3}:\left[0, l_{3}\right] \longrightarrow X_{n}$ corresponding to the sides $[b, c]$ and $[c, a]$ respectively.

Observe that if $\alpha_{1}\left(l_{1}\right) \neq \alpha_{2}(0)$, then both points belong to some $\eta_{n m}$, since we have a treedecomposition; condition $(b)$ gives that $d_{X_{n}}\left(\alpha_{1}\left(l_{1}\right), \alpha_{2}(0)\right) \leq k_{1}$. The same is true if $\alpha_{2}\left(l_{2}\right) \neq \alpha_{3}(0)$, and if $\alpha_{3}\left(l_{3}\right) \neq \alpha_{1}(0)$. Hence $T_{n}:=\alpha_{1} \cup \alpha_{2} \cup \alpha_{3}$ is a $\left(2 \max \left\{1, k_{2}\right\}, 2 k_{1}+2 k_{3}, k_{1}\right)$-quasigeodesic triangle. Lemma 4 gives that $T_{n}$ is $\delta_{1}$-thin, with $\delta_{1}=k_{1}+4 k_{4}+2 H\left(k_{4}, 2 \max \left\{1, k_{2}\right\}, 4 k_{1}+2 k_{3}\right)$, where $H$ is the constant in Theorem C.

If $z \in X_{n}$, without loss of generality we can assume that $z \in \alpha_{1}$; if $A^{\prime}:=\alpha_{2} \cup \alpha_{3}$, then there exists $z^{\prime} \in A^{\prime}$ with $d_{X_{n}}\left(z, A^{\prime}\right)=d_{X_{n}}\left(z, z^{\prime}\right) \leq \delta_{1}$. If $z^{\prime} \in A$, then $d_{X}(z, A) \leq \delta_{1}$. If $z^{\prime} \notin A$, then, there exists $z_{0} \in A$ such that $d_{X_{n}}\left(z_{0}, z^{\prime}\right) \leq k_{1} / 2$; then, $d_{X}(z, A) \leq d_{X_{n}}\left(z, z^{\prime}\right)+d_{X_{n}}\left(z^{\prime}, z_{0}\right) \leq \delta_{1}+k_{1} / 2$.

If only two sides of $T$ intersect $X_{n}$, we have the same result since we can see a bigon as a triangle with two equal vertices. These facts prove Corollary 1 . We finish now the proof of Theorem 1.

If $A \cap X_{n}=\varnothing$, then $z$ belongs to some geodesic $g_{0} \subseteq g$ joining some $\eta_{m k}$ with itself such that $A \cap$ $X_{m} \neq \varnothing$, since we have a tree-decomposition. By $(b)$, there exists $z^{\prime} \in g_{0} \cap \eta_{m k}$ with $d_{X}\left(z, z^{\prime}\right) \leq k_{1} / 2$, and then, there exists $z_{0} \in A \cap X_{m}$ such that $d_{X_{m}}\left(z_{0}, z^{\prime}\right) \leq \delta_{1}+k_{1} / 2$. Consequently, $d_{X}(z, A) \leq \delta_{1}+k_{1}$, and $X$ is $\delta$-thin with $\delta:=2 k_{1}+4 k_{4}+2 H\left(k_{4}, 2 \max \left\{1, k_{2}\right\}, 4 k_{1}+2 k_{3}\right)$.

Let us assume that $X$ is $\delta$-hyperbolic.

We prove now that the inclusion $i: X_{n} \longrightarrow X$ is a $\left(2 \max \left\{1, k_{2}\right\}, 2 k_{1}+2 k_{3}\right)$-quasi-isometry.

Given $x, y \in X_{n}$, we have that $d_{X}(x, y) \leq d_{X_{n}}(x, y)$, since there are more curves joining $x$ and $y$ in $X$ than in $X_{n}$. In order to prove the other inequality, let us consider a geodesic $g$ in $X$ joining $x$ and $y$. If $g \subseteq X_{n}$, then $d_{X}(x, y)=d_{X_{n}}(x, y)$. In other case, we can define $t_{1}^{1}, t_{1}^{2}, \ldots, t_{r}^{1}, t_{r}^{2}, s_{1}^{1}, s_{1}^{2}, \ldots, s_{j}^{1}, s_{j}^{2}$, and the $\left(2 \max \left\{1, k_{2}\right\}, 2 k_{1}+2 k_{3}\right)$-quasigeodesic $\alpha_{1}:\left[0, l_{1}\right] \longrightarrow X_{n}$ joining $x$ and $y$ as in the proof of the first part of the theorem. Since $\alpha_{1}$ has its arc-length parametrization, $\frac{1}{2} \min \left\{1, k_{2}^{-1}\right\} L\left(\alpha_{1}\right)-2\left(k_{1}+k_{3}\right) \leq$ $d_{X}\left(\alpha_{1}(0), \alpha_{1}\left(l_{1}\right)\right)=d_{X}(x, y)$.

Since $\alpha_{1}$ is a continuous curve in $X_{n}$ joining $x$ and $y, d_{X_{n}}(x, y) \leq L\left(\alpha_{1}\right)$, and then

$$
\frac{1}{2} \min \left\{1, k_{2}^{-1}\right\} d_{X_{n}}(x, y)-2\left(k_{1}+k_{3}\right) \leq d_{X}(x, y) \leq d_{X_{n}}(x, y) .
$$

Hence, if $X$ is $\delta$-hyperbolic, then $X_{n}$ is $4 \max \left\{1, k_{2}\right\}\left(2 \delta+k_{1}+k_{3}+H\left(\delta, 2 \max \left\{1, k_{2}\right\}, 2 k_{1}+2 k_{3}\right)\right)$-thin (see [GH, p.88]).

Let us observe that in this proof of the hyperbolicity of $X_{n}$ we do not use that the other pieces are tree-pieces; this gives Corollary 2.

Theorem 2 below let us move the study of the hyperbolicity of a certain space $X$ to another space $Y$ with simpler structure, so long as between them there exists the type of relationship described by the following definition. 
Definition 11. We say that two geodesic metric spaces $X$ and $Y$ (in this order) have comparable decompositions, if there exist decompositions $\left\{X_{n}\right\}_{n \in \Lambda}$ of $X$ and $\left\{Y_{n}\right\}_{n \in \Lambda}$ of $Y$, and constants $k_{i}$, with the following properties:

(a) If $X_{n} \cap X_{m}=\cup_{i \in I_{n m}} \eta_{n m}^{i}$, then $Y_{n} \cap Y_{m}=\cup_{i \in I_{n m}} \sigma_{n m}^{i}$, and $\sigma_{n m}^{i}=\varnothing$ if and only if $\eta_{n m}^{i}=\varnothing$.

(b) For any $n, m, i, \operatorname{diam}_{X_{n}}\left(\eta_{n m}^{i}\right) \leq k_{1}$ and $\operatorname{diam}_{Y_{n}}\left(\sigma_{n m}^{i}\right) \leq k_{1}$.

(c) We can split $\Lambda$ into $F \cup G$ and $F$ into $F_{1} \cup F_{2}$ with:

(c1) If $n \in G, X_{n}$ is a $\left(k_{1}, k_{2}, k_{3}\right)$-tree-piece.

(c2) If $n \in F, \operatorname{diam}_{X_{n}}\left(\eta_{n m}^{i}\right) \leq k_{2} d_{X_{n}}\left(\eta_{n m}^{i}, \eta_{n k}^{j}\right)$ and $\operatorname{diam}_{Y_{n}}\left(\sigma_{n m}^{i}\right) \leq k_{2} d_{Y_{n}}\left(\sigma_{n m}^{i}, \sigma_{n k}^{j}\right)$ if $(m, i) \neq$ $(k, j)$.

(c3) If $n \in F_{1}$, for each $\eta_{n m}^{i} \neq \eta_{n k}^{j}$, there exists a geodesic $\gamma_{m n k}^{i j}$ in $X_{n}$, joining $\eta_{n m}^{i}$ with $\eta_{n k}^{j}$, and a $\left(k_{4}, b_{m n k}^{i j}\right)$-quasi-isometry $f_{m n k}^{i j}: \gamma_{m n k}^{i j} \longrightarrow h_{m n k}^{i j} \subseteq Y_{n}$, with $h_{m n k}^{i j}$ starting in $\sigma_{n m}^{i}$ and finishing in $\sigma_{n k}^{j}$, and $\sum_{n \in F_{1}} \sum_{m, k, i, j} b_{m n k}^{i j} \leq k_{5}$, such that for any $x, y \in \cup_{m, k, i, j} \gamma_{m n k}^{i j}$, with corresponding points $x^{\prime}, y^{\prime} \in \cup_{m, k, i, j} h_{m n k}^{i j}$, we have $k_{4}^{-1} d_{X_{n}}(x, y)-k_{5} \leq d_{Y_{n}}\left(x^{\prime}, y^{\prime}\right)$.

(c4) If $n \in F_{2}$, there exists a $\left(k_{4}, 0\right)$-quasi-isometry $f_{n}: X_{n} \longrightarrow Y_{n}$, with $f_{n}\left(\eta_{n m}^{i}\right) \subseteq \sigma_{n m}^{i}$.

\section{Remarks.}

1. Obviously, these conditions are required only for $\eta_{n m}^{i}, \sigma_{n m}^{i} \neq \varnothing$.

2. The sets $\Lambda, F, G$ and $I_{n m}$ do not need to be countable.

3. We obviously have $\eta_{n m}^{i}=\eta_{m n}^{i}$ and $I_{n m}=I_{m n}$.

4. The hypothesis $(c 2)$ trivially holds if for $n \in F, d_{X_{n}}\left(\eta_{n m}^{i}, \eta_{n k}^{j}\right) \geq k_{2}^{\prime}$ and $d_{Y_{n}}\left(\sigma_{n m}^{i}, \sigma_{n k}^{j}\right) \geq k_{2}^{\prime}$, by $(b)$.

5. The hypothesis $(c 3)$ can be relaxed: let us consider any connected component $B_{s}$ of $\cup_{n \in F} X_{n}$; the proof of Theorem 2 gives that it is enough to have $\sum_{n \in F_{1}^{s}, m, k, i, j} b_{m n k}^{i j} \leq k_{5}$, for any $s$, where $F_{1}^{s}:=\left\{n \in F_{1}: X_{n} \subseteq B_{s}\right\}$ (see the construction of $T_{2}$ in the proof of Theorem 2).

6. As a consequence of $(c 3)$, we have that $k_{4}^{-1} d_{X_{n}}\left(x, \eta_{n r}^{t}\right)-k_{1}-k_{5} \leq d_{Y_{n}}\left(f_{m n k}^{i j}(x), \sigma_{n r}^{t}\right)$, for every $x \in \gamma_{m n k}^{i j}$ and $r, t$.

7. Since condition $(c 3)$ can be tedious to check, it could be interesting to check instead the following statement which implies $(c 3)$ :

$\left(c 3^{\prime}\right)$ If $n \in F_{1}$, we have that $k_{7}^{-1} \leq d_{X_{n}}\left(\eta_{n m}^{i}, \eta_{n k}^{j}\right) / d_{Y_{n}}\left(\sigma_{n m}^{i}, \sigma_{n k}^{j}\right) \leq k_{7}, \operatorname{diam}_{X_{n}}\left(\cup_{m i} \eta_{n m}^{i}\right) \leq k_{8}$ and $\operatorname{diam}_{Y_{n}}\left(\cup_{m i} \sigma_{n m}^{i}\right) \leq k_{8}$.

In the decomposition of $X$ one can find pieces of two different types: $\left\{X_{n}\right\}_{n \in F}$ and $\left\{X_{n}\right\}_{n \in G}$. The connections among a piece $X_{n}$, with $n \in G$, and the rest of the pieces are simple enough for being $X_{n}$ a tree-piece. The connections of the pieces $X_{n}$, with $n \in F$, do not have topological restrictions; therefore, besides ( $b$ ) and $(c 2)$ (as in the case $n \in G$ ), they must be controlled somehow: the conditions $(c 3)$ and $(c 4)$ let us assure that the connections between $X_{n}$ and the rest of the pieces must be alike to the ones in $Y_{n}$. Observe that condition (c3) involves just a small subset of points of each $X_{n}$, with $n \in F_{1}$.

In spite of lengthening Definition 11, splitting $\Lambda$ into the union of the three types of sets $F_{1}, F_{2}$ and $G$ is an extremely convenient course of action: on the one hand, the wider the range of possibilities, the easier it will be to fit a certain piece into one of them. On the other hand, the determination of 
the conditions that $X_{n}$ must verify when $n$ belongs to $F_{1}, F_{2}$ or $G$, is not arbitrary at all. In fact, what lies behind is an appropriate modelization for the study of the following problem in Riemann surfaces (see [PRT]): Given a Riemann surface $S$, another one $S^{*}$ can be obtained from $S$ by removing a union of simply connected closed sets $\left\{E_{m}\right\}_{m \in M}$. In [PRT] it is proved that $S$ is hyperbolic if and only if $S^{*}$ is hyperbolic, when $\left\{E_{m}\right\}$ are sufficiently separated. Theorem 2 is used in the proof of the latest statement: The idea is to consider some neighborhoods of $\left\{E_{m}\right\}$ as pieces $\left\{S_{m}\right\}$ (in $S^{*}$ we take $\left.S_{m}^{*}:=S_{m} \backslash E_{m}\right)$. $G$ is defined as the set of $m$ 's belonging to $M$ such that $S_{m}$ is a tree-piece; $F_{1}$ is the rest of indices of $M$, and $F_{2}$ is the set of indices which parametrizes the connected components of $S \backslash \cup_{m \in M} S_{m}$ (in $S^{*}$ we take the same connected components). Finally, Definition 11 has been formulated by abstracting the essential properties of pieces in each of the three sets.

Theorem 2. Let us assume that two geodesic metric spaces $X$ and $Y$ have comparable decompositions. If $Y$ is $\delta^{\prime}$-hyperbolic and there exists a constant $k_{6}$ such that $X_{n}$ is $k_{6}$-hyperbolic for every $n \in \Lambda \backslash F_{2}$, then $X$ is $\delta$-hyperbolic, with $\delta$ a constant which only depends on $\delta^{\prime}$ and $k_{i}$.

There is an explicit expression of $\delta$ at the end of the proof of Theorem 2 .

It is obvious that $(c 4)$ is much more restrictive than $(c 3)$; however, it is a small price to pay in return for not having to check the hyperbolicity of pieces in $F_{2}$.

We can see this theorem as a version of Theorem B: if $\Lambda=F_{1}$, condition $(c 3)$ says somehow that there is a quasi-isometry of a small subset of $X$ on a subset of $Y$. From a dual point of view, we can consider that there is a quasi-isometry of a subset of $Y$ on a subset of $X$; in this case we have the surprising result that the hyperbolicity of the original space implies the hyperbolicity of the final space.

The hyperbolicity of $X$ does not imply the uniform hyperbolicity of $X_{n}$ in general (this is another difference with Theorem 1). In fact, the hyperbolicity of $X$ does not guarantee the hyperbolicity of each $X_{n}$, as it is shown in the following example: let us consider $X_{1}$ as the Cayley graph of the group $\mathbf{Z}^{2}$, and $X_{2}$ the tree with a countable number of edges of length 1 with a common vertex $v_{0}$; we construct $X$ by gluing in a bijective way each vertex of $X_{2}$ (except for $v_{0}$ ) with a vertex of $X_{1}$; it is clear that $X$ is hyperbolic since it is bounded, and that $X_{1}$ is not hyperbolic. In the same line, it is easy to construct a locally finite graph $X=\cup_{n} X_{n}$ with $\lim _{n \rightarrow \infty} \delta\left(X_{n}\right)=\infty$.

Next, we provide some conditions which guarantee the hyperbolicity of $X_{n}$.

Proposition 1. Let $X$ be a $\delta$-hyperbolic geodesic metric space with a decomposition as in Definition 11. If for some $n \in \Lambda$ there exist constants $k_{7} \geq 1, k_{8} \geq 0$, with $d_{X_{n}}\left(\eta_{n m}^{i}, \eta_{n k}^{j}\right) \leq k_{7} d_{X}\left(\eta_{n m}^{i}, \eta_{n k}^{j}\right)+k_{8}$, for any $m, k, i, j$, then $X_{n}$ is $k_{6}$-hyperbolic, with $k_{6}:=4 k_{7}\left(4 \delta+k_{7}^{-1}\left(2 k_{1}+k_{8}\right)+2 H\left(\delta, k_{7}, k_{7}^{-1}\left(2 k_{1}+k_{8}\right)\right)\right)$, where $H$ is the constant in Theorem $C$.

The following result is weaker than the one in Proposition 1, but it has the advantage that it only involves distances in $X_{n}$. In fact, this is the best possible general result involving just distances in $X_{n}$; besides it allows to get sharper constants.

Proposition 2. Let $X$ be a $\delta$-hyperbolic geodesic metric space with a decomposition as in Definition 11. If for some $n \in \Lambda$ there exists a positive constant $k_{7}$ with $\operatorname{diam}_{X_{n}}\left(\cup_{m i} \eta_{n m}^{i}\right) \leq k_{7}$, then $X_{n}$ is 
$\left(\delta+3 k_{7} / 2\right)$-hyperbolic.

Corollary 3. Let us assume that two geodesic metric spaces $X$ and $Y$ have comparable decompositions, that $Y$ is hyperbolic, and that there exists a positive constant $k_{7}$ with $\operatorname{diam}_{X_{n}}\left(\cup_{m i} \eta_{n m}^{i}\right) \leq k_{7}$ for every $n \in \Lambda$. Then $X$ is hyperbolic if and only if there exists a constant $k_{6}$ such that $X_{n}$ is $k_{6}$-hyperbolic for every $n \in \Lambda$.

Proof of Theorem 2. Let us consider a geodesic triangle $T=\{a, b, c\}$ in $X$. It is obvious that if $T \subseteq X_{n}$ for some $n \in \Lambda \backslash F_{2}$, then $T$ is $4 k_{6}$-thin by hypothesis. In other case (i.e., whether $T \subseteq X_{n}$ with $n \in F_{2}$ or $T$ intersects several $X_{n}$ 's), the main idea of the proof is to choose successively quasigeodesic triangles $T_{1}, T_{2}, T_{3}, T_{4}$ in $X$ (closely related to $T$ ), which will allow to construct a quasigeodesic triangle $T_{5}$ in $Y$ (related to $T_{4}$ ). Since $Y$ is hyperbolic, then $T_{5}$ is thin by Lemma 4 , and we will use this information in order to obtain that $T$ is also thin. One of the main obstacles is that although $X$ and $Y$ have similar connections among their components, each pair of spaces $X_{n}$ and $Y_{n}$ can be very different (in fact, a quasi-isometry might not exist between $X_{n}$ and $Y_{n}$ ).

Even though the main idea is simple, the proof is long and technical; in order to make the arguments more transparent, we collect some results we need along the proof in technical lemmas. Most of them will be proved in the last section of the paper.

A partial goal is to obtain a triangle $T_{4}$ in $X$ easily transformable into another triangle $T_{5}$ in $Y$ (in fact, $T \cap X_{n}$ is contained in $\cup_{m, k, i, j} \gamma_{m n k}^{i j}$ if $n \in \Lambda \backslash F_{2}$ ). In order to do this, the first step is to obtain a triangle $T_{1}$ in $X$ such that for any $n \in \Lambda \backslash F_{2}$, each connected component of $T_{1} \cap X_{n}$ is a geodesic in $X_{n}$. Recall that although each connected component of $S \cap X_{n}$ of any side $S$ of $T$ is a geodesic in $X_{n}$, there can exist connected components of $T \cap X_{n}$ (containing a vertex of $T$ ) which are not geodesics in $X_{n}$.

We start with the construction of $T_{1}$.

Let us assume that in the piece $X_{n}$ there is at least one vertex $a$ of $T$. If $n \in F_{2}$, we do not change $T \cap X_{n}$. (In particular, if $T \subseteq X_{n}$, with $n \in F_{2}$, then $T_{1}=T$.) Let us consider now $n \in \Lambda \backslash F_{2}$, and let us call $\eta_{a}$ to the connected component of $T \cap X_{n}$ which contains $a$.

Case 1. Assume first that $\eta_{a}$ only contains a vertex of $T$. We denote by $x_{1}, x_{2}$ the end points of $\eta_{a}$. Then, we consider a geodesic triangle $T_{a}=\left\{a, x_{1}, x_{2}\right\}$ in $X_{n}$ with $\left[a, x_{1}\right],\left[a, x_{2}\right] \subset T$. Let us denote by $a_{1}$ the internal point of $T_{a}$ in the geodesic $\left[x_{1}, x_{2}\right]$ in $X_{n}$. We define $\eta_{a_{1}}:=\left[x_{1}, x_{2}\right]=$ $\left[x_{1}, a_{1}\right] \cup\left[a_{1}, x_{2}\right] \subset T_{a}$. If $b \in X_{m}$ (where $m$ can be either $n$ or not) and the connected component $\eta_{b}$ of $T \cap X_{m}$ which contains $b$ does not contain $c$, then we can proceed with the vertices $b, c$ in a similar way that with $a$. In this case, $T_{1}$ is defined as the (not necessarily geodesic) triangle connecting the vertices $a_{1}, b_{1}, c_{1}$, obtained from $T$ by replacing $\eta_{a}, \eta_{b}, \eta_{c}$ by $\eta_{a_{1}}, \eta_{b_{1}}, \eta_{c_{1}}$ respectively.

Case 2. Let us assume now that $b \in \eta_{a}$ and $c \notin \eta_{a}$.

Without loss of generality, we can assume that $\eta_{a}$ starts in $x_{1}$, ends in $x_{2}$, and meets $a$ before than $b$. We consider the quadrilateral $\eta_{a} \cup\left[x_{1}, x_{2}\right] \subseteq X_{n}$ and we draw its diagonal $\left[a, x_{2}\right]$ (we can get a similar result by drawing $\left[b, x_{1}\right]$ ), obtaining two geodesic triangles in $X_{n}: T_{a}=\left\{a, x_{1}, x_{2}\right\}$ (with the internal points $u_{1} \in\left[a, x_{2}\right], u_{2} \in\left[x_{1}, x_{2}\right]$ and $u_{3} \in\left[a, x_{1}\right]$ ), $T_{b}=\left\{a, b, x_{2}\right\}$ (with the internal points $v_{1} \in\left[a, x_{2}\right], v_{2} \in\left[b, x_{2}\right]$ and $\left.v_{3} \in[a, b]\right)$. 
Case 2.1. We consider first the situation $d_{X_{n}}\left(x_{2}, v_{1}\right)<d_{X_{n}}\left(x_{2}, u_{1}\right)$. We denote by $b_{1}$ the point in $\left[x_{1}, x_{2}\right]$ with $d_{X_{n}}\left(x_{2}, b_{1}\right)=d_{X_{n}}\left(x_{2}, v_{1}\right)$. If we denote $a_{1}:=u_{2}$, we can define $\eta_{a_{1}}:=\left[x_{1}, x_{2}\right]=$ $\left[x_{1}, a_{1}\right] \cup\left[a_{1}, b_{1}\right] \cup\left[b_{1}, x_{2}\right] \subset T_{a}$. We define $\eta_{c_{1}}$ as in Case 1 . Then we construct the triangle $T_{1}$ connecting the vertices $a_{1}, b_{1}, c_{1}$, obtained from $T$ by replacing $\eta_{a}, \eta_{c}$ by $\eta_{a_{1}}, \eta_{c_{1}}$ respectively.

Case 2.2. We consider the situation $d_{X_{n}}\left(x_{2}, v_{1}\right) \geq d_{X_{n}}\left(x_{2}, u_{1}\right)$. If we denote $a_{1}:=b_{1}:=u_{2}$, we can define $\eta_{a_{1}}:=\left[x_{1}, x_{2}\right]=\left[x_{1}, a_{1}\right] \cup\left[a_{1}, x_{2}\right] \subset T_{a}$. We define $\eta_{c_{1}}$ as in Case 1 . Then we construct the bigon $T_{1}$ connecting the vertices $a_{1}, c_{1}$, obtained from $T$ by replacing $\eta_{a}, \eta_{c}$ by $\eta_{a_{1}}, \eta_{c_{1}}$ respectively.

Case 3. Finally, let us assume that $b, c \in \eta_{a}$. Without loss of generality, we can assume that $\eta_{a}$ starts in $x_{1}$, ends in $x_{2}$, and meets $a$ before than $b$ and meets $b$ before than $c$.

We consider the pentagon $\eta_{a} \cup\left[x_{1}, x_{2}\right] \subseteq X_{n}$ and we draw its diagonals $\left[x_{1}, b\right],\left[b, x_{2}\right]$, obtaining three geodesic triangles in $X_{n}: T_{a}=\left\{a, b, x_{1}\right\}$ (with the internal points $s_{1} \in[a, b], s_{2} \in\left[a, x_{1}\right], s_{3} \in\left[b, x_{1}\right]$ ), $T_{b}=\left\{b, x_{1}, x_{2}\right\}$ (with the internal points $u_{1} \in\left[b, x_{2}\right], u_{2} \in\left[b, x_{1}\right]$ and $u_{3} \in\left[x_{1}, x_{2}\right]$ ), $T_{c}=\left\{b, c, x_{2}\right\}$ (with the internal points $v_{1} \in\left[c, x_{2}\right], v_{2} \in[b, c]$ and $v_{3} \in\left[b, x_{2}\right]$ ).

Case 3.1. We consider first the situation $d_{X_{n}}\left(x_{1}, s_{3}\right)<d_{X_{n}}\left(x_{1}, u_{2}\right)$ and $d_{X_{n}}\left(x_{2}, v_{3}\right)<d_{X_{n}}\left(x_{2}, u_{1}\right)$. We denote by $a_{1}$ the point in $\left[x_{1}, x_{2}\right]$ with $d_{X_{n}}\left(x_{1}, a_{1}\right)=d_{X_{n}}\left(x_{1}, s_{3}\right)$, and by $c_{1}$ the point in $\left[x_{1}, x_{2}\right]$ with $d_{X_{n}}\left(x_{2}, c_{1}\right)=d_{X_{n}}\left(x_{2}, v_{3}\right)$. If we denote $b_{1}:=u_{3}$, we can define $\eta_{a_{1}}:=\left[x_{1}, x_{2}\right]=\left[x_{1}, a_{1}\right] \cup$ $\left[a_{1}, b_{1}\right] \cup\left[b_{1}, c_{1}\right] \cup\left[c_{1}, x_{2}\right] \subset T_{b}$. Then we construct the triangle $T_{1}$ connecting the vertices $a_{1}, b_{1}, c_{1}$, obtained from $T$ by replacing $\eta_{a}$ by $\eta_{a_{1}}$.

Case 3.2. We consider now the situation $d_{X_{n}}\left(x_{1}, s_{3}\right) \geq d_{X_{n}}\left(x_{1}, u_{2}\right)$ and $d_{X_{n}}\left(x_{2}, v_{3}\right)<d_{X_{n}}\left(x_{2}, u_{1}\right)$. We define $a_{1}:=b_{1}:=u_{3}$, and we denote by $c_{1}$ the point in $\left[x_{1}, x_{2}\right]$ with $d_{X_{n}}\left(x_{2}, c_{1}\right)=d_{X_{n}}\left(x_{2}, v_{3}\right)$. We can define $\eta_{a_{1}}:=\left[x_{1}, x_{2}\right]=\left[x_{1}, a_{1}\right] \cup\left[a_{1}, c_{1}\right] \cup\left[c_{1}, x_{2}\right] \subset T_{b}$. Then we construct the bigon $T_{1}$ connecting the vertices $a_{1}, c_{1}$, obtained from $T$ by replacing $\eta_{a}$ by $\eta_{a_{1}}$.

Case 3.3. The situation $d_{X_{n}}\left(x_{1}, s_{3}\right)<d_{X_{n}}\left(x_{1}, u_{2}\right)$ and $d_{X_{n}}\left(x_{2}, v_{3}\right) \geq d_{X_{n}}\left(x_{2}, u_{1}\right)$ is symmetric to Case 3.2 , changing the roles of $a$ and $c$.

Case 3.4. Finally, we consider the situation $d_{X_{n}}\left(x_{1}, s_{3}\right) \geq d_{X_{n}}\left(x_{1}, u_{2}\right)$ and $d_{X_{n}}\left(x_{2}, v_{3}\right) \geq d_{X_{n}}\left(x_{2}, u_{1}\right)$. In this case, we do not construct the triangle $T_{1}$.

Lemma 5. If $T_{1}$ is $\delta_{1}$-thin, then $T$ is $\delta_{0}$-thin, with $\delta_{0}:=\max \left\{\delta_{1}+16 k_{6}, 18 k_{6}\right\}$.

See the proof of Lemma 5 in Section 3.

We have the following elementary fact.

Lemma 6. Let us consider a metric space $X$, an interval $I$, an $(a, b)$-quasigeodesic $g: I \longrightarrow X$ and a curve $g_{1}: I \longrightarrow X$ such that $d\left(g(t), g_{1}(t)\right) \leq c$ for every $t \in I$. Then $g_{1}$ is $a(a, b+2 c)$-quasigeodesic.

Proof. For any $s, t \in I$, we have that

$$
\begin{aligned}
& d\left(g_{1}(t), g_{1}(s)\right) \leq d\left(g_{1}(t), g(t)\right)+d(g(t), g(s))+d\left(g(s), g_{1}(s)\right) \leq a|t-s|+b+2 c, \\
& d\left(g_{1}(t), g_{1}(s)\right) \geq d(g(t), g(s))-d\left(g_{1}(t), g(t)\right)-d\left(g(s), g_{1}(s)\right) \geq a^{-1}|t-s|-b-2 c .
\end{aligned}
$$

Lemma 7. Each side of $T_{1}$ is a $\left(1,16 k_{6}\right)$-quasigeodesic with its arc-length parametrization. Furthermore, each connected component of $T_{1} \cap X_{n}$ is a geodesic in $X_{n}$, if $n \in \Lambda \backslash F_{2}$. 
See the proof of Lemma 7 in Section 3.

As a second step, we split the triangle $T_{1}$ in several parts; Corollary 1 will allow to forget the part of $T_{1}$ which intersects the pieces $X_{n}$ with $n \in G$ (see Lemma 8).

We consider the connected components $\left\{B_{s}\right\}_{s \in S}$ of the set $\cup_{n \in F} X_{n}$. We can study the triangle $T_{1}$ in each piece of $\left\{B_{s}\right\}_{s \in S}$ and of $\left\{X_{n}\right\}_{n \in G}$. We denote by $T_{2}$ the quasigeodesic triangle $T_{1} \cap B_{s}$, for some fixed $s \in S$; in fact, we should write $T_{2}^{s}$ instead of $T_{2}$, but our notation is simpler and there will be no place to confusion. Let us observe that $T_{2}$ is the union of three sides (possibly not continuous) joining $a_{2}$ with $b_{2}^{\prime}, b_{2}$ with $c_{2}^{\prime}$ and $c_{2}$ with $a_{2}^{\prime}$.

Recall that we want to obtain a triangle $T_{4}$ in $X$ contained in $\cup_{n, m, k, i, j} \gamma_{m n k}^{i j}$. As a third step, we construct the triangle $T_{3}$ in order to remove from $T_{2}$ the connected components of $T_{2} \cap X_{n}$ which join some $\eta_{n m}^{i}$ with itself.

We define the triangle $T_{3}$ in the following way:

Without loss of generality we can consider a side $g_{1}$ of $T_{1}$ as the oriented curve from $a_{1}$ to $b_{1}$. We have that $a_{2}=g_{1}(\alpha)$ and $b_{2}^{\prime}=g_{1}(\beta)$, for some real numbers $\alpha<\beta$. By hypothesis, $g_{1}$ meets at most a finite number of $\eta_{n m}^{i}$ 's. Let us assume that $g_{1}$ meets $\cup_{n, m, i} \eta_{n m}^{i}$. As we consider $g_{1}:[\alpha, \beta] \longrightarrow X$, let us define

$$
t_{1}^{1}:=\min \left\{\alpha \leq t \leq \beta: g_{1}(t) \in \cup_{n, m, i} \eta_{n m}^{i}\right\}
$$

There exists this minimum since $g_{1}$ is a continuous function in a compact interval and $g_{1} \cap\left(\cup_{n, m, i} \eta_{n m}^{i}\right)$ is a compact set: each $\eta_{n m}^{i}$ is a closed set and $g_{1}$ meets at most a finite number of $\eta_{n m}^{i}$ 's.

Then $g_{1}\left(t_{1}^{1}\right) \in \eta_{n_{1} m_{1}}^{i_{1}}$, for some $n_{1}, m_{1}, i_{1}$, and we define

$$
t_{1}^{2}:=\max \left\{\alpha \leq t \leq \beta: g_{1}(t) \in \eta_{n_{1} m_{1}}^{i_{1}}\right\}
$$

In a similar way, we define recursively

$$
t_{j}^{1}:=\min \left\{t_{j-1}^{2}<t \leq \beta: g_{1}(t) \in \cup_{n, m, i} \eta_{n m}^{i}\right\}
$$

if $g_{1}\left(t_{j}^{1}\right) \in \eta_{n_{j} m_{j}}^{i_{j}}$, for some $n_{j}, m_{j}, i_{j}$, we take

$$
t_{j}^{2}:=\max \left\{t_{j-1}^{2}<t \leq \beta: g_{1}(t) \in \eta_{n_{j} m_{j}}^{i_{j}}\right\}
$$

We can continue this choice for $1 \leq j \leq r$. We define $t_{0}^{2}:=\alpha$ if $\alpha \neq t_{1}^{1}$, and $t_{r+1}^{1}:=\beta$ if $\beta \neq t_{r}^{2}$.

We define $g_{3}$ (in this case) as the restriction of $g_{1}$ to the set $\left[\alpha, t_{1}^{1}\right] \cup\left(t_{1}^{2}, t_{2}^{1}\right] \cup \cdots \cup\left(t_{r-1}^{2}, t_{r}^{1}\right] \cup\left(t_{r}^{2}, \beta\right]$. If $g_{1}$ does not intersects $\cup_{n, m, i} \eta_{n m}^{i}$, we take $g_{3}=g_{1}$. We define $a_{3}:=a_{2}$ if $\alpha<t_{1}^{1}$ and $a_{3}:=g_{1}\left(t_{1}^{2}\right)$ if $\alpha=t_{1}^{1}$; we define $b_{3}^{\prime}:=b_{2}^{\prime}$ if $t_{r}^{2}<\beta$ and $b_{3}^{\prime}:=g_{1}\left(t_{r}^{1}\right)$ if $t_{r}^{2}=\beta . g_{3}$ is a left continuous curve between $a_{3}$ and $b_{3}^{\prime}$. We consider a similar construction with the other sides of $T_{2}$. The triangle $T_{3}$ is the union of these three curves.

Lemma 8. If $T_{3}$ is $\delta_{3}$-thin, then $T_{1}$ is $\max \left\{\delta_{3}+k_{1}, \delta^{*}\right\}$-thin, with $\delta^{*}:=3 k_{1} / 2+4 k_{6}+2 H\left(k_{6}, 2 \max \left\{1, k_{2}\right\}, 4 k_{1}+\right.$ $\left.2 k_{3}\right)$, where $H$ is the constant in Theorem $C$. 
Proof. We study the triangle $T_{1}$ in each piece of $\left\{B_{s}\right\}_{s \in S}$ and of $\left\{X_{n}\right\}_{n \in G}$.

Recall that $(c 1)$ gives that for any $n \in G, X_{n}$ is a $\left(k_{1}, k_{2}, k_{3}\right)$-tree-piece. Corollary 1 gives that $T_{3} \cap X_{n}$ is $\delta^{*}$-thin for every $n \in G$ (we can assume that $X_{n}$ intersects at least two sides of $T_{3}$; if $X_{n}$ had intersected only one side of $T_{1}$, this part of $T_{1}$ would have been removed during the construction of $T_{3}$, since $X_{n}$ is a tree-piece). We consider now $T_{1} \cap B_{s}$ for each $s$.

By (b) and the construction of $T_{3}$, given any $z \in T_{1} \cap B_{s}$, there exists $z_{2} \in T_{3}$ in the corresponding side of $z$, with $d_{X}\left(z, z_{2}\right) \leq d_{X_{n}}\left(z, z_{2}\right) \leq k_{1}$. Then there exists $w$ in the union of the two other sides of $T_{3}$ with $d_{X}\left(w, z_{2}\right) \leq \delta_{3}$. Since $T_{3} \subseteq T_{1} \cap B_{s}$, we have the result.

Lemma 9. Each side of $T_{3}$ is a $\left(1+k_{2}, k_{1}+16 k_{6}\right)$-quasigeodesic with its arc-length parametrization. Furthermore, each connected component of $T_{3} \cap X_{n}$ is a geodesic in $X_{n}$, if $n \in F_{1}$.

See the proof of Lemma 9 in Section 3.

Remark. After the construction of $T_{3}$ and lemmas 8 and 9 , without loss of generality we can assume that there is a unique component $B_{s}$, i.e. that $T_{3}$ is a $\left(1+k_{2}, k_{1}+16 k_{6}, k_{1}\right)$-quasigeodesic triangle in $X$, with $\Lambda=F$ and $G=\varnothing$.

We construct the triangle $T_{4}$ by changing each geodesic segment in $T_{3}$ joining $\eta_{n m}^{i}$ with $\eta_{n k}^{j}$ by a new geodesic $\gamma_{m n k}^{i j}$. This triangle and conditions $(c 3)$ and $(c 4)$ will allow to obtain a triangle $T_{5}$ in $Y$ in an obvious way.

These are the details in the construction of $T_{4}$ :

Each connected component of $T_{3}$ is a geodesic segment $g_{m n k}^{i j}$ in some $X_{n}$, joining $\eta_{n m}^{i}$ with $\eta_{n k}^{j}$. If $n \in F_{1},(c 3)$ gives that for each $g_{m n k}^{i j}$ there exists a geodesic $\gamma_{m n k}^{i j}$ in $X_{n}$, joining $\eta_{n m}^{i}$ with $\eta_{n k}^{j}$, and a $\left(k_{4}, b_{m n k}^{i j}\right)$-quasi-isometry $f_{m n k}^{i j}: \gamma_{m n k}^{i j} \longrightarrow h_{m n k}^{i j} \subseteq Y_{n}$. If $n \in F_{2}$, we define $f_{m n k}^{i j}$ as the restriction of $f_{n}$ to $g_{m n k}^{i j}, \gamma_{m n k}^{i j}:=g_{m n k}^{i j}$, and $h_{m n k}^{i j}:=f_{m n k}^{i j}\left(\gamma_{m n k}^{i j}\right)$. (Then, $f_{m n k}^{i j}$ is a $\left(k_{4}, b_{m n k}^{i j}\right)$-quasi-isometry, with $b_{m n k}^{i j}:=0$.)

We obtain $T_{4}$ in $X$ by replacing each $g_{m n k}^{i j}$ by $\gamma_{m n k}^{i j}$. We only need to choose the vertices of $T_{4}$, if some vertex of $T_{3}$ is in $\cup_{n \in F_{1}} X_{n}$ :

Let us consider $n \in F_{1}$ and the arc-length parametrizations $g_{m n k}^{i j}:[0, l] \longrightarrow X$ and $\gamma_{m n k}^{i j}:\left[0, l^{\prime}\right] \longrightarrow$ $X$. We observe first that $(c 2)$ gives $l^{\prime}-l=L_{X}\left(\gamma_{m n k}^{i j}\right)-L_{X}\left(g_{m n k}^{i j}\right) \leq \operatorname{diam}_{X_{n}}\left(\eta_{n m}^{i}\right)+\operatorname{diam}_{X_{n}}\left(\eta_{n k}^{j}\right) \leq$ $2 k_{2} L_{X}\left(g_{m n k}^{i j}\right)=2 k_{2} l$. Therefore we conclude $l^{\prime} / l \leq 1+2 k_{2}$, and symmetrically $l / l^{\prime} \leq 1+2 k_{2}$.

Lemma 10. Let us consider an absolute continuous and bijective function between two intervals $u: I \longrightarrow J$ with $c^{-1} \leq\left|u^{\prime}\right| \leq c$, and an $(a, b)$-quasigeodesic $g: J \longrightarrow X$. Then $g \circ u: I \longrightarrow X$ is an $(a c, b)$-quasigeodesic.

Proof. We have that $c^{-1}|t-s| \leq|u(t)-u(s)| \leq c|t-s|$, and hence $a^{-1} c^{-1}|t-s|-b \leq a^{-1}|u(t)-u(s)|-b \leq d(g(u(t)), g(u(s))) \leq a|u(t)-u(s)|+b \leq a c|t-s|+b$.

Lemma 11. Let us consider two geodesics $\gamma_{1}:\left[0, l_{1}\right] \longrightarrow X$ and $\gamma_{2}:\left[0, l_{2}\right] \longrightarrow X$ in a $\delta$-fine space $X$, with $d\left(\gamma_{1}(0), \gamma_{2}(0)\right) \leq c$ and $d\left(\gamma_{1}\left(l_{1}\right), \gamma_{2}\left(l_{2}\right)\right) \leq c$. Then $d\left(\gamma_{1}(t), \gamma_{2}\left(l_{2} t / l_{1}\right)\right) \leq 2 \delta+7 c$, for $t \in\left[0, l_{1}\right]$.

See the proof of Lemma 11 in Section 3. 
We consider the reparametrization $g_{m n k}^{i j}\left(l t / l^{\prime}\right):\left[0, l^{\prime}\right] \longrightarrow X$ of $g_{m n k}^{i j}$; recall that $l^{\prime} / l, l / l^{\prime} \leq 1+$ $2 k_{2}$. Using these local reparametrizations, if $G_{3}: J_{0} \longrightarrow X$ and $G_{4}: I_{0} \longrightarrow X$ are arc-length parametrizations of $T_{3}$ and $T_{4}$ (respectively), we can construct a global bijection $u: I_{0} \longrightarrow J_{0}$ (in fact, a continuous juxtaposition of straight lines) with $\left(1+2 k_{2}\right)^{-1} \leq\left|u^{\prime}\right| \leq 1+2 k_{2}$. Since $G_{3} \circ u$ and $G_{4}$ are defined over the same interval $I_{0}$, if $\left(G_{3} \circ u\right)\left(t_{0}\right)$ is a vertex in $T_{3}$, for some $t_{0} \in I_{0}$, we can define $G_{4}\left(t_{0}\right)$ as its corresponding vertex in $T_{4}$. Lemmas 9 and 10 give that if $g_{3}=\left.\left(G_{3} \circ u\right)\right|_{I}$, is a side of $T_{3}$, for some interval $I$, then $g_{3}$ is a $\left(\left(1+k_{2}\right)\left(1+2 k_{2}\right), k_{1}+16 k_{6}\right)$-quasigeodesic. Observe that $g_{4}:=\left.G_{4}\right|_{I}$ is an arc-length parametrization of the side of $T_{4}$ corresponding to $g_{3}$. Since we have $(b)$, Lemma 11 gives that $d_{X}\left(g_{3}(t), g_{4}(t)\right) \leq 8 k_{6}+7 k_{1}$, for every $t \in I$. Then, Lemma 6 implies that $g_{4}$ is a $\left(\left(1+k_{2}\right)\left(1+2 k_{2}\right), 15 k_{1}+32 k_{6}\right)$-quasigeodesic. Consequently we obtain the following result.

Lemma 12. Each side of $T_{4}$ is a $\left(\left(1+k_{2}\right)\left(1+2 k_{2}\right), 15 k_{1}+32 k_{6}\right)$-quasigeodesic with its arc-length parametrization. Furthermore, each connected component of $T_{4} \cap X_{n}$ is a geodesic in $X_{n}$, if $n \in F_{1}$. If $T_{4}$ is $\delta_{4}$-thin, then $T_{3}$ is $\left(\delta_{4}+14 k_{1}+16 k_{6}\right)$-thin.

Proof. We have proved the first two statements. In order to prove the last one we only need to remark that for every point in any side of $T_{3}$ there is another one in the corresponding side of $T_{4}$ which is at distance $7 k_{1}+8 k_{6}$ at most; the same result is true if we change the roles of $T_{3}$ and $T_{4}$.

Let us observe that if $T \subseteq X_{n}$, with $n \in F_{2}$, then $T_{4}=T$.

So far, we have modified the original triangle in $X$ to obtain a new one $T_{4}$ which can now be easily transformed into a triangle $T_{5}$ in $Y$ by replacing $\gamma_{m n k}^{i j} \subseteq X_{n}$ by $h_{m n k}^{i j} \subseteq Y_{n}$. We take the canonical parametrization $f_{m n k}^{i j}\left(\gamma_{m n k}^{i j}(t)\right)$ in $h_{m n k}^{i j}$, where $t$ is the arc-length parameter for $\gamma_{m n k}^{i j}$.

Lemma 13. Each side of $T_{5}$ is a $\left(d_{1}, d_{2}\right)$-quasigeodesic with its canonical parametrization, where $d_{0}:=\left(1+k_{2}\right)\left(1+2 k_{2}\right) k_{4}, d_{1}:=d_{0}\left(1+k_{2}\right)\left(1+2 k_{2}\right)$ and

$$
d_{2}:=\max \left\{k_{1}+\left(1+k_{2}\right) k_{5}, k_{4}\left(15 k_{1}+32 k_{6}\right)+k_{5}, d_{0}^{-1}\left(17 k_{1}+32 k_{6}\right)+2\left(k_{1}+k_{5}\right)+\left(1+2 k_{2}\right)^{-1} k_{5}\right\} .
$$

In fact, the proof of Lemma 13 (see Section 3) gives the following result.

Corollary 4. For any $x, y \in T_{4}$ with corresponding points $x^{\prime}, y^{\prime} \in T_{5}$, we have that $d_{X}(x, y) \leq$ $d_{0} d_{Y}\left(x^{\prime}, y^{\prime}\right)+2 k_{1}+d_{0}\left(2\left(k_{1}+k_{5}\right)+\left(1+2 k_{2}\right)^{-1} k_{5}\right)$.

By Lemma 13, the sides of $T_{5}$ are $\left(d_{1}, d_{2}\right)$-quasigeodesics. By $(b)$ and the construction of $T_{5}$, we have that an end point of any side of $T_{5}$ has an end point of another side at distance less or equal than $k_{1}$. Since $Y$ is $\delta^{\prime}$-hyperbolic, Lemma 4 gives that $T_{5}$ is $\delta_{5}$-thin with $\delta_{5}:=4 \delta^{\prime}+k_{1}+2 H\left(\delta^{\prime}, d_{1}, d_{2}+2 k_{1}\right)$. Now Corollary 4 gives that $T_{4}$ is $\delta_{4}$-thin, with $\delta_{4}:=d_{0} \delta_{5}+2 k_{1}+d_{0}\left(2\left(k_{1}+k_{5}\right)+\left(1+2 k_{2}\right)^{-1} k_{5}\right)$.

Lemma 12 gives that $T_{3}$ is $\delta_{3}$-thin with $\delta_{3}:=\delta_{4}+14 k_{1}+16 k_{6}$. By Lemma 8 , we have that $T_{1}$ is $\delta_{1}$-thin with $\delta_{1}:=\max \left\{\delta_{3}+k_{1}, \delta^{*}\right\}$, where $\delta^{*}=3 k_{1} / 2+4 k_{6}+2 H\left(k_{6}, 2 \max \left\{1, k_{2}\right\}, 4 k_{1}+2 k_{3}\right)$. Theorem 2 is now a consequence of Lemma 5 , and we have $\delta:=4\left(\delta_{1}+16 k_{6}\right)$, since $\delta_{1} \geq 2 k_{6}$ (in fact, $\left.\delta_{1} \geq \delta_{3} \geq 16 k_{6}\right)$. 
Proof of Proposition 1. Firstly we prove that the inclusion $i: X_{n} \longrightarrow X$ is a $\left(k_{7}, k_{7}^{-1}\left(2 k_{1}+k_{8}\right)\right)$ quasi-isometry.

Given $x, y \in X_{n}$, we have that $d_{X}(x, y) \leq d_{X_{n}}(x, y)$, since there are more curves joining $x$ and $y$ in $X$ than in $X_{n}$. In order to prove the other inequality, let us consider a geodesic $g$ in $X$ joining $x$ and $y$. If $g \subseteq X_{n}$, then $d_{X}(x, y)=d_{X_{n}}(x, y)$. In other case, we have for some $m, k, i, j$,

$$
\begin{aligned}
& d_{X}(x, y) \geq d_{X_{n}}\left(x, \eta_{n m}^{i}\right)+d_{X}\left(\eta_{n m}^{i}, \eta_{n k}^{j}\right)+d_{X_{n}}\left(y, \eta_{n k}^{j}\right) \\
& \quad \geq d_{X_{n}}\left(x, \eta_{n m}^{i}\right)+k_{7}^{-1} d_{X_{n}}\left(\eta_{n m}^{i}, \eta_{n k}^{j}\right)+d_{X_{n}}\left(y, \eta_{n k}^{j}\right)-k_{8} k_{7}^{-1} \\
& \quad \geq k_{7}^{-1}\left(d_{X_{n}}\left(x, \eta_{n m}^{i}\right)+\operatorname{diam}_{X_{n}}\left(\eta_{n m}^{i}\right)+d_{X_{n}}\left(\eta_{n m}^{i}, \eta_{n k}^{j}\right)+\operatorname{diam}_{X_{n}}\left(\eta_{n k}^{j}\right)+d_{X_{n}}\left(y, \eta_{n k}^{j}\right)-2 k_{1}-k_{8}\right) \\
& \quad \geq k_{7}^{-1} d_{X_{n}}(x, y)-k_{7}^{-1}\left(2 k_{1}+k_{8}\right) .
\end{aligned}
$$

Hence, since $X$ is $\delta$-hyperbolic, then $X_{n}$ is $k_{7}\left(4 \delta+k_{7}^{-1}\left(2 k_{1}+k_{8}\right)+2 H\left(\delta, k_{7}, k_{7}^{-1}\left(2 k_{1}+k_{8}\right)\right)\right)$-thin (see [GH, p.88]).

Proof of Proposition 2. Given $x, y \in X_{n}$, we have that

$$
d_{X}(x, y) \leq d_{X_{n}}(x, y) \leq d_{X}(x, y)+\operatorname{diam}_{X_{n}}\left(\cup_{m i} \eta_{n m}^{i}\right) \leq d_{X}(x, y)+k_{7} .
$$

If we denote by $(x, y)_{w}$ and $(x, y)_{w, n}$ the Gromov products in $X$ and $X_{n}$ respectively, the last inequalities give for any $x, y, w \in X_{n}$

$$
(x, y)_{w, n}-k_{7} \leq(x, y)_{w} \leq(x, y)_{w, n}+k_{7} / 2 .
$$

Then, we deduce for any $x, y, z, w \in X_{n}$, that

$$
\begin{aligned}
(x, z)_{w, n} & \geq(x, z)_{w}-k_{7} / 2 \geq \min \left\{(x, y)_{w},(y, z)_{w}\right\}-\delta-k_{7} / 2 \\
& \geq \min \left\{(x, y)_{w, n}-k_{7},(y, z)_{w, n}-k_{7}\right\}-\delta-k_{7} / 2 \geq \min \left\{(x, y)_{w, n},(y, z)_{w, n}\right\}-\delta-3 k_{7} / 2 .
\end{aligned}
$$

Hence, $X_{n}$ is $\left(\delta+3 k_{7} / 2\right)$-hyperbolic.

\section{§3. Proof of technical lemmas}

Lemma 5. For each point $z$ in one side of $T$, we denote by $A=A(z)$ the union of the two other sides of $T$. If we are in case 3.4 we have $d_{X}(z, A) \leq 18 k_{6}$. In other case, we have either:

(1) $d_{X}(z, A) \leq 12 k_{6}$, or

(2) there exists a point $z_{1} \in T_{1}$ with $d_{X}\left(z, z_{1}\right) \leq 8 k_{6}$, and besides $z$ and $z_{1}$ are in corresponding sides.

Moreover, for each point $z_{1}$ in one side of $T_{1}$ there exists a point $z \in T$ with $d_{X}\left(z, z_{1}\right) \leq 8 k_{6}$, and furthermore $z$ and $z_{1}$ are in corresponding sides.

Consequently, if $T_{1}$ is $\delta_{1}$-thin, then $T$ is $\delta_{0}$-thin, with $\delta_{0}:=\max \left\{\delta_{1}+16 k_{6}, 18 k_{6}\right\}$.

Proof. Recall that if $n \in F_{2}$, then $T_{1} \cap X_{n}=T \cap X_{n}$. Consequently, we can assume that the vertices of $T$ belong to $\cup_{n \in \Lambda \backslash F_{2}} X_{n}$, since in other case the argument is easier.

If $z \notin \eta_{a} \cup \eta_{b} \cup \eta_{c}$, then $z \in T_{1}$ and we have (2) with $z_{1}=z$. In other case we can assume that $z \in \eta_{a}$. We consider now the same cases in the construction of $T_{1}$ in the proof of Theorem 2. 
Case 1. We have that $\eta_{a} \subseteq X_{n}$ only contains a vertex of $T$. Let us denote by $a_{1}, x_{1}^{\prime}, x_{2}^{\prime}$ the internal points of the geodesics $\left[x_{1}, x_{2}\right],\left[a, x_{2}\right],\left[a, x_{1}\right]$ in $X_{n}$ respectively. We have $\eta_{a_{1}}:=\left[x_{1}, x_{2}\right]=$ $\left[x_{1}, a_{1}\right] \cup\left[a_{1}, x_{2}\right] \subset T_{a}$. Since $T_{a}$ is $4 k_{6}$-fine in $X_{n}$ by the hypothesis and Theorem A, if $z \in\left[x_{1}, x_{2}^{\prime}\right]$ then there exists $z_{1} \in\left[x_{1}, a_{1}\right]$ with $d_{X}\left(z_{1}, z\right) \leq d_{X_{n}}\left(z_{1}, z\right) \leq 4 k_{6}$, and if $z \in\left[x_{1}^{\prime}, x_{2}\right]$ then there exists $z_{1} \in\left[a_{1}, x_{2}\right]$ with $d_{X}\left(z_{1}, z\right) \leq 4 k_{6}$; then, we have (2). If $z \in\left[a, x_{1}^{\prime}\right]$, we can take $w \in\left[a, x_{2}^{\prime}\right]$ with $d_{X}(z, w) \leq 4 k_{6} ; z \in\left[a, x_{2}^{\prime}\right]$, we can take $w \in\left[a, x_{1}^{\prime}\right]$ with $d_{X}(z, w) \leq 4 k_{6}$; then, we have (1).

Case 2. We have now that $b \in \eta_{a}$ and $c \notin \eta_{a}$.

Case 2.1. We consider the situation $d_{X_{n}}\left(x_{2}, v_{1}\right)<d_{X_{n}}\left(x_{2}, u_{1}\right)$. We denote by $u_{1}^{\prime}$ the point in $\left[a, v_{3}\right] \subset[a, b]$ with $d_{X_{n}}\left(a, u_{1}\right)=d_{X_{n}}\left(a, u_{1}^{\prime}\right)$.

(i) If $z \in\left[x_{1}, u_{3}\right] \subseteq\left[x_{1}, a\right]$, then there exists $z_{1} \in\left[x_{1}, a_{1}\right]$ such that $d_{X}\left(z, z_{1}\right) \leq 4 k_{6}$.

(ii) If $z \in\left[u_{1}^{\prime}, v_{3}\right]$, then there exists $z_{1} \in\left[a_{1}, b_{1}\right]$ such that $d_{X}\left(z, z_{1}\right) \leq 8 k_{6}$, since the triangles $T_{a}$ and $T_{b}$ are $4 k_{6}$-fine.

(iii) If $z \in\left[x_{2}, v_{2}\right] \subseteq\left[x_{2}, b\right]$, then there exists $z_{1} \in\left[b_{1}, x_{2}\right]$ with $d_{X}\left(z, z_{1}\right) \leq 8 k_{6}$.

In these three cases we have (2).

(iv) If $z \in\left[a, u_{3}\right]$, then there exists $w \in\left[a, u_{1}^{\prime}\right]$ such that $d_{X}(z, w) \leq 8 k_{6}$.

(v) If $z \in\left[a, u_{1}^{\prime}\right]$, then there exists $w \in\left[a, u_{3}\right]$ such that $d_{X}(z, w) \leq 8 k_{6}$.

(vi) If $z \in\left[b, v_{3}\right]$, then there exists $w \in\left[b, v_{2}\right]$ such that $d_{X}(z, w) \leq 4 k_{6}$.

(vii) If $z \in\left[b, v_{2}\right]$, then there exists $w \in\left[b, v_{3}\right]$ such that $d_{X}(z, w) \leq 4 k_{6}$.

In these four cases we have (1).

Case 2.2. We consider the situation $d_{X_{n}}\left(x_{2}, v_{1}\right) \geq d_{X_{n}}\left(x_{2}, u_{1}\right)$. Let us recall that $a_{1}=b_{1}$. We denote by $v_{1}^{\prime}$ the point in $\left[a, u_{3}\right] \subset\left[a, x_{1}\right]$ with $d_{X_{n}}\left(a, v_{1}\right)=d_{X_{n}}\left(a, v_{1}^{\prime}\right)$ and by $u_{1}^{\prime}$ the point in $\left[v_{2}, x_{2}\right] \subset\left[b, x_{2}\right]$ with $d_{X_{n}}\left(x_{2}, u_{1}\right)=d_{X_{n}}\left(x_{2}, u_{1}^{\prime}\right)$.

(i) If $z \in\left[x_{1}, u_{3}\right] \subseteq\left[x_{1}, a\right]$, then there exists $z_{1} \in\left[x_{1}, a_{1}\right]$ such that $d_{X}\left(z, z_{1}\right) \leq 4 k_{6}$.

(ii) If $z \in\left[x_{2}, u_{1}^{\prime}\right] \subseteq\left[x_{2}, b\right]$, then there exists $z_{1} \in\left[b_{1}, x_{2}\right]$ with $d_{X}\left(z, z_{1}\right) \leq 8 k_{6}$.

In these two cases we have (2).

(iii) If $z \in\left[a, v_{1}^{\prime}\right]$, then there exists $w \in\left[a, v_{3}\right]$ such that $d_{X}(z, w) \leq 8 k_{6}$.

(iv) If $z \in\left[a, v_{3}\right]$, then there exists $w \in\left[a, v_{1}^{\prime}\right]$ such that $d_{X}(z, w) \leq 8 k_{6}$.

(v) If $z \in\left[b, v_{3}\right]$, then there exists $w \in\left[b, v_{2}\right]$ such that $d_{X}(z, w) \leq 4 k_{6}$.

(vi) If $z \in\left[b, v_{2}\right]$, then there exists $w \in\left[b, v_{3}\right]$ such that $d_{X}(z, w) \leq 4 k_{6}$.

(vii) If $z \in\left[u_{3}, v_{1}^{\prime}\right]$, then there exists $w \in\left[v_{2}, u_{1}^{\prime}\right] \subset\left[b, u_{1}^{\prime}\right]$ such that $d_{X}(z, w) \leq 8 k_{6}$.

(viii) If $z \in\left[v_{2}, u_{1}^{\prime}\right]$, then there exists $w \in\left[v_{1}^{\prime}, u_{3}\right] \subset\left[a, u_{3}\right]$ such that $d_{X}(z, w) \leq 8 k_{6}$.

In these five cases we have (1).

Case 3. We have now that $b, c \in \eta_{a}$.

Case 3.1. We consider the situation $d_{X_{n}}\left(x_{1}, s_{3}\right)<d_{X_{n}}\left(x_{1}, u_{2}\right)$ and $d_{X_{n}}\left(x_{2}, v_{3}\right)<d_{X_{n}}\left(x_{2}, u_{1}\right)$. We denote by $u_{2}^{\prime}$ the point in $\left[b, s_{1}\right] \subset[a, b]$ with $d_{X_{n}}\left(b, u_{2}\right)=d_{X_{n}}\left(b, u_{2}^{\prime}\right)$, and by $u_{1}^{\prime}$ the point in $\left[b, v_{2}\right] \subset[b, c]$ with $d_{X_{n}}\left(b, u_{1}\right)=d_{X_{n}}\left(b, u_{1}^{\prime}\right)$.

(i) If $z \in\left[x_{1}, s_{2}\right] \subseteq\left[x_{1}, a\right]$, then there exists $z_{1} \in\left[x_{1}, a_{1}\right]$ such that $d_{X}\left(z, z_{1}\right) \leq 8 k_{6}$, since the triangles $T_{a}$ and $T_{b}$ are $4 k_{6}$-fine.

(ii) If $z \in\left[s_{1}, u_{2}^{\prime}\right]$, then there exists $z_{1} \in\left[a_{1}, b_{1}\right]$ such that $d_{X}\left(z, z_{1}\right) \leq 8 k_{6}$. 
(iii) If $z \in\left[u_{1}^{\prime}, v_{2}\right]$, then there exists $z_{1} \in\left[b_{1}, c_{1}\right]$ such that $d_{X}\left(z, z_{1}\right) \leq 8 k_{6}$.

(iv) If $z \in\left[x_{2}, v_{1}\right] \subseteq\left[x_{2}, c\right]$, then there exists $z_{1} \in\left[c_{1}, x_{2}\right]$ with $d_{X}\left(z, z_{1}\right) \leq 8 k_{6}$.

In these four cases we have (2).

(v) If $z \in\left[a, s_{2}\right]$, then there exists $w \in\left[a, s_{1}\right]$ such that $d_{X}(z, w) \leq 4 k_{6}$. We have a similar result if $z \in\left[a, s_{1}\right]$.

(vi) If $z \in\left[b, u_{2}^{\prime}\right]$, then there exists $w \in\left[b, u_{1}^{\prime}\right]$ such that $d_{X}(z, w) \leq 12 k_{6}$. We have a similar result if $z \in\left[b, u_{1}^{\prime}\right]$.

(vii) If $z \in\left[c, v_{2}\right]$, then there exists $w \in\left[c, v_{1}\right]$ such that $d_{X}(z, w) \leq 4 k_{6}$. We have a similar result if $z \in\left[c, v_{1}\right]$.

In these three cases we have (1).

Case 3.2. We have the situation $d_{X_{n}}\left(x_{1}, s_{3}\right) \geq d_{X_{n}}\left(x_{1}, u_{2}\right)$ and $d_{X_{n}}\left(x_{2}, v_{3}\right)<d_{X_{n}}\left(x_{2}, u_{1}\right)$. We denote by $u_{2}^{\prime}$ the point in $\left[x_{1}, s_{2}\right] \subset\left[x_{1}, a\right]$ with $d_{X_{n}}\left(x_{1}, u_{2}\right)=d_{X_{n}}\left(x_{1}, u_{2}^{\prime}\right)$, by $u_{1}^{\prime}$ the point in $\left[b, v_{2}\right] \subset$ $[b, c]$ with $d_{X_{n}}\left(b, u_{1}\right)=d_{X_{n}}\left(b, u_{1}^{\prime}\right)$, and by $s_{3}^{\prime}$ the point in $\left[b, v_{2}\right] \subset[b, c]$ with $d_{X_{n}}\left(b, s_{3}\right)=d_{X_{n}}\left(b, s_{3}^{\prime}\right)$.

(i) If $z \in\left[x_{1}, u_{2}^{\prime}\right] \subseteq[a, c]$, then there exists $z_{1} \in\left[x_{1}, a_{1}\right]$ such that $d_{X}\left(z, z_{1}\right) \leq 8 k_{6}$.

(ii) If $z \in\left[u_{1}^{\prime}, v_{2}\right] \subseteq[b, c]$, then there exists $z_{1} \in\left[b_{1}, c_{1}\right]$ such that $d_{X}\left(z, z_{1}\right) \leq 8 k_{6}$.

(iii) If $z \in\left[x_{2}, v_{1}\right] \subseteq[a, c]$, then there exists $z_{1} \in\left[c_{1}, x_{2}\right]$ with $d_{X}\left(z, z_{1}\right) \leq 8 k_{6}$.

In these three cases we have (2).

(iv) If $z \in\left[a, s_{2}\right]$, then there exists $w \in\left[a, s_{1}\right]$ such that $d_{X}(z, w) \leq 4 k_{6}$. We have a similar result if $z \in\left[a, s_{1}\right]$.

(v) If $z \in\left[b, s_{1}\right]$, then there exists $w \in\left[b, s_{3}^{\prime}\right]$ such that $d_{X}(z, w) \leq 12 k_{6}$. We have a similar result if $z \in\left[b, s_{3}^{\prime}\right]$.

(vi) If $z \in\left[c, v_{2}\right]$, then there exists $w \in\left[c, v_{1}\right]$ such that $d_{X}(z, w) \leq 4 k_{6}$. We have a similar result if $z \in\left[c, v_{1}\right]$.

(vii) If $z \in\left[u_{2}^{\prime}, s_{2}\right]$, then there exists $w \in\left[u_{1}^{\prime}, s_{3}^{\prime}\right]$ such that $d_{X}(z, w) \leq 12 k_{6}$. We have a similar result if $z \in\left[u_{1}^{\prime}, s_{3}^{\prime}\right]$.

In these four cases we have (1).

Case 3.3 is similar to 3.2 .

Case 3.4. We have the situation $d_{X_{n}}\left(x_{1}, s_{3}\right) \geq d_{X_{n}}\left(x_{1}, u_{2}\right)$ and $d_{X_{n}}\left(x_{2}, v_{3}\right) \geq d_{X_{n}}\left(x_{2}, u_{1}\right)$. Without loss of generality we can assume that $d_{X_{n}}\left(b, v_{3}\right) \geq d_{X_{n}}\left(b, s_{3}\right)$, since the other case is similar. We denote by $v_{3}^{\prime}$ the point in $\left[b, u_{2}\right] \subset\left[b, x_{1}\right]$ with $d_{X_{n}}\left(b, v_{3}\right)=d_{X_{n}}\left(b, v_{3}^{\prime}\right)$, by $v_{3}^{\prime \prime}$ the point in $\left[x_{1}, s_{2}\right] \subset\left[x_{1}, a\right]$ with $d_{X_{n}}\left(x_{1}, v_{3}^{\prime}\right)=d_{X_{n}}\left(x_{1}, v_{3}^{\prime \prime}\right)$, and by $s_{1}^{\prime}$ the point in $\left[b, v_{2}\right] \subseteq[b, c]$ with $d_{X_{n}}\left(b, s_{1}\right)=d_{X_{n}}\left(b, s_{1}^{\prime}\right)$.

(i) If $z \in\left[a, s_{2}\right]$, then there exists $w \in\left[a, s_{1}\right]$ such that $d_{X}(z, w) \leq 4 k_{6}$. We have a similar result if $z \in\left[a, s_{1}\right]$.

(ii) If $z \in\left[b, s_{1}\right]$, then there exists $w \in\left[b, s_{1}^{\prime}\right]$ such that $d_{X}(z, w) \leq 12 k_{6}$. We have a similar result if $z \in\left[b, s_{1}^{\prime}\right]$.

(iii) If $z \in\left[c, v_{2}\right]$, then there exists $w \in\left[c, v_{1}\right]$ such that $d_{X}(z, w) \leq 4 k_{6}$. We have a similar result if $z \in\left[c, v_{1}\right]$.

(iv) If $z \in\left[v_{3}^{\prime \prime}, s_{2}\right]$, then there exists $w \in\left[v_{2}, s_{1}^{\prime}\right]$ such that $d_{X}(z, w) \leq 12 k_{6}$. We have a similar result if $z \in\left[v_{2}, s_{1}^{\prime}\right]$. 
(v) In other case, $z \in\left[v_{3}^{\prime \prime}, v_{1}\right] \subseteq[a, c]$. We have that $L_{X}\left(\left[v_{3}^{\prime \prime}, v_{1}\right]\right)=d_{X}\left(v_{3}^{\prime \prime}, v_{1}\right) \leq 12 k_{6}$; consequently $d_{X}\left(z,\left\{v_{3}^{\prime \prime}, v_{1}\right\}\right) \leq 6 k_{6}$ and $d_{X}(z, A) \leq 6 k_{6}+12 k_{6}=18 k_{6}$.

This finishes the proof of the first part of the lemma. The proof of the second one follows a similar argument and is easier, since there is no dichotomy.

Finally, let us see that $T_{1}$ is $\delta_{1}$-thin in $X$ implies that $T$ is $\delta_{0}$-thin in $X$. We consider $z \in T$; if $z$ satisfies (1), there is nothing to prove. In other case, there exists $z_{1} \in T_{1}$ such that $d_{X}\left(z, z_{1}\right) \leq 8 k_{6}$ and $z$ and $z_{1}$ are in corresponding sides. Since $T_{1}$ is $\delta_{1}$-thin in $X$, there exists $w_{1} \in T_{1}$ with $d_{X}\left(z_{1}, w_{1}\right) \leq \delta_{1}$ and $w_{1}$ in the union of the two other sides. The second part of the lemma gives that there exists $w \in A$ with $d_{X}\left(w_{1}, w\right) \leq 8 k_{6}$. Therefore $d_{X}(z, A) \leq d_{X}(z, w) \leq \delta_{1}+16 k_{6}$.

Lemma 7. Each side of $T_{1}$ is a $\left(1,16 k_{6}\right)$-quasigeodesic with its arc-length parametrization. Furthermore, each connected component of $T_{1} \cap X_{n}$ is a geodesic in $X_{n}$, if $n \in \Lambda \backslash F_{2}$.

Proof. We can assume that the vertices of $T$ belong to $\cup_{n \in \Lambda \backslash F_{2}} X_{n}$, since in other case the argument is easier.

The second statement is a direct consequence of the construction of $T_{1}$. This first one is a consequence of Lemma 6 and the construction of $T_{1}$ :

If $g: J \longrightarrow X$ is a geodesic side of $T$, Lemma 6 gives that it is enough to check that there exists a subinterval $I \subseteq J$ such that $g_{1}: I \longrightarrow X$ is the arc-length parametrization for the corresponding side in $T_{1}$ of $g$, and that $d_{X}\left(g(t), g_{1}(t)\right) \leq 8 k_{6}$ for every $t \in I$.

We consider now the same cases in the construction of $T_{1}$ in the proof of Theorem 2.

Case 1. If $\left[x_{1}, x_{2}^{\prime}\right] \subset g$, then we substitute this interval for $\left[x_{1}, a_{1}\right]$ in order to obtain $g_{1}$, and then we have $d_{X}\left(g(t), g_{1}(t)\right) \leq 4 k_{6}$ in these $\operatorname{arcs}$, since $T_{a}$ is $4 k_{6}$-fine. The case $\left[x_{2}, x_{1}^{\prime}\right] \subset g$ is similar.

Case 2. If $\left[x_{1}, u_{3}\right] \subset g$, then we substitute this interval for $\left[x_{1}, a_{1}\right]$ in order to obtain $g_{1}$, and then we have $d_{X}\left(g(t), g_{1}(t)\right) \leq 4 k_{6}$ in these arcs. The case $\left[x_{2}, v_{2}\right] \subset g$ is similar, with constant $8 k_{6}$, since $T_{a}$ and $T_{b}$ are $4 k_{6}$-fine.

Case 2.1. If $g=[a, b]$, then $\left[u_{1}^{\prime}, v_{3}\right] \subset g$ and $g_{1}=\left[a_{1}, b_{1}\right]$. We have $d_{X}\left(g(t), g_{1}(t)\right) \leq 8 k_{6}$ in $g_{1}$.

Case 2.2. If $g=[a, b]$, then $a_{1}=b_{1}$ and $g_{1}$ is this unique point.

Case 3.1. If $\left[x_{1}, s_{2}\right] \subset g$, then we substitute this interval for $\left[x_{1}, a_{1}\right]$ in order to obtain $g_{1}$, and then we have $d_{X}\left(g(t), g_{1}(t)\right) \leq 8 k_{6}$ in these arcs. The case $\left[x_{2}, v_{1}\right] \subset g$ is similar.

If $g=[a, b]$, then $\left[s_{1}, u_{2}^{\prime}\right] \subset g$ and $g_{1}=\left[a_{1}, b_{1}\right]$. We have $d_{X}\left(g(t), g_{1}(t)\right) \leq 8 k_{6}$ in $g_{1}$. If $g=[b, c]$, then $\left[u_{1}^{\prime}, v_{2}\right] \subset g$ and $g_{1}=\left[b_{1}, c_{1}\right]$. We have $d_{X}\left(g(t), g_{1}(t)\right) \leq 8 k_{6}$ in $g_{1}$.

Case 3.2. If $g=[a, c]$, we have $\left[x_{1}, u_{2}^{\prime}\right] \cup\left[x_{2}, v_{1}\right] \subset g$, and then we substitute these intervals for $\left[x_{1}, a_{1}\right] \cup\left[x_{2}, c_{1}\right]$ (respectively) in order to obtain $g_{1}$; then we have $d_{X}\left(g(t), g_{1}(t)\right) \leq 8 k_{6}$ in these arcs.

If $g=[b, c]$, then $\left[u_{1}^{\prime}, v_{2}\right] \subset g$ and $g_{1}=\left[b_{1}, c_{1}\right]$. We have $d_{X}\left(g(t), g_{1}(t)\right) \leq 8 k_{6}$ in $g_{1}$.

If $g=[a, b]$, then $a_{1}=b_{1}$ and $g_{1}$ is this unique point.

Case 3.3 is similar to 3.2 ; we do not consider 3.4 since in this case we do not have $T_{1}$.

Lemma 9. Each side of $T_{3}$ is a $\left(1+k_{2}, k_{1}+16 k_{6}\right)$-quasigeodesic with its arc-length parametrization. Furthermore, each connected component of $T_{3} \cap X_{n}$ is a geodesic in $X_{n}$, if $n \in F_{1}$. 
Proof. We can assume that the vertices of $T$ belong to $\cup_{n \in F_{1}} X_{n}$, since in other case the argument is easier.

The second statement is a direct consequence of the construction of $T_{3}$ and Lemma 7 . In order to see the first one, let us consider an arc-length parametrization $g_{1}:[0, l] \longrightarrow X$ of one side of $T_{1}$. Without loss of generality we can assume that $g_{1}(0)=a_{2}$ and $g_{1}(l)=b_{2}^{\prime} . \quad g_{1}$ is a $\left(1,16 k_{6}\right)^{-}$ quasigeodesic by Lemma 7 . We consider now an arc-length parametrization $g_{3}:\left[0, l^{\prime}\right] \longrightarrow X$ of the side of $T_{3}$ corresponding to $g_{1}$. If $g_{3}=g_{1}$, there is nothing to prove.

In other case, if $s, t \in\left[0, l^{\prime}\right]$ there exist $s^{*} \in\left(t_{i-1}^{2}, t_{i}^{1}\right]$ and $t^{*} \in\left(t_{j-1}^{2}, t_{j}^{1}\right]$ such that $s=s^{*}-\sum_{k=1}^{i-1}\left(t_{k}^{2}-\right.$ $\left.t_{k}^{1}\right), t=t^{*}-\sum_{k=1}^{j-1}\left(t_{k}^{2}-t_{k}^{1}\right), g_{3}(s)=g_{1}\left(s^{*}\right)$ and $g_{3}(t)=g_{1}\left(t^{*}\right)$. Provided that $i=j$, we have that

$$
\begin{aligned}
& d_{X}\left(g_{3}(t), g_{3}(s)\right)=d_{X}\left(g_{1}\left(t^{*}\right), g_{1}\left(s^{*}\right)\right) \leq\left|t^{*}-s^{*}\right|=|t-s|, \\
& d_{X}\left(g_{3}(t), g_{3}(s)\right)=d_{X}\left(g_{1}\left(t^{*}\right), g_{1}\left(s^{*}\right)\right) \geq\left|t^{*}-s^{*}\right|-16 k_{6}=|t-s|-16 k_{6} .
\end{aligned}
$$

Otherwise, we can assume that $i<j$. Then we have that

$$
\begin{aligned}
& d_{X}\left(g_{3}(t), g_{3}(s)\right)=d_{X}\left(g_{1}\left(t^{*}\right), g_{1}\left(s^{*}\right)\right) \leq t^{*}-s^{*}=t-s+\sum_{k=i}^{j-1}\left(t_{k}^{2}-t_{k}^{1}\right), \\
& d_{X}\left(g_{3}(t), g_{3}(s)\right)=d_{X}\left(g_{1}\left(t^{*}\right), g_{1}\left(s^{*}\right)\right) \geq t^{*}-s^{*}-16 k_{6} \geq t-s-16 k_{6} .
\end{aligned}
$$

Observe that $(c 2)$ gives $t_{k+1}^{1}-t_{k}^{2} \geq k_{2}^{-1}\left(t_{k}^{2}-t_{k}^{1}\right)$. This fact implies that

$$
t-s \geq \sum_{k=i}^{j-2}\left(t_{k+1}^{1}-t_{k}^{2}\right) \geq k_{2}^{-1} \sum_{k=i}^{j-2}\left(t_{k}^{2}-t_{k}^{1}\right) .
$$

This inequality and $(b)$ give

$$
d_{X}\left(g_{3}(t), g_{3}(s)\right) \leq t-s+\sum_{k=i}^{j-2}\left(t_{k}^{2}-t_{k}^{1}\right)+t_{j-1}^{2}-t_{j-1}^{1} \leq\left(1+k_{2}\right)(t-s)+k_{1} .
$$

Lemma 11. Let us consider two geodesics $\gamma_{1}:\left[0, l_{1}\right] \longrightarrow X$ and $\gamma_{2}:\left[0, l_{2}\right] \longrightarrow X$ in a $\delta$-fine space $X$, with $d\left(\gamma_{1}(0), \gamma_{2}(0)\right) \leq c$ and $d\left(\gamma_{1}\left(l_{1}\right), \gamma_{2}\left(l_{2}\right)\right) \leq c$. Then $d\left(\gamma_{1}(t), \gamma_{2}\left(l_{2} t / l_{1}\right)\right) \leq 2 \delta+7 c$, for $t \in\left[0, l_{1}\right]$.

Proof. Without loss of generality we can assume that $l_{1} \leq l_{2}$. We consider the geodesic quadrilateral $Q=\left\{\gamma_{1}(0), \gamma_{1}\left(l_{1}\right), \gamma_{2}\left(l_{2}\right), \gamma_{2}(0)\right\}$ and the geodesic triangles $T_{1}=\left\{\gamma_{1}(0), \gamma_{1}\left(l_{1}\right), \gamma_{2}(0)\right\}$ (with internal points $p_{1} \in \gamma_{1}, p_{2} \in\left[\gamma_{1}\left(l_{1}\right), \gamma_{2}(0)\right], p_{3} \in\left[\gamma_{1}(0), \gamma_{2}(0)\right]$ ) and $T_{3}=\left\{\gamma_{1}\left(l_{1}\right), \gamma_{2}\left(l_{2}\right), \gamma_{2}(0)\right\}$ (with internal points $\left.q_{1} \in\left[\gamma_{1}\left(l_{1}\right), \gamma_{2}(0)\right], q_{2} \in \gamma_{2}, q_{3} \in\left[\gamma_{1}\left(l_{1}\right), \gamma_{2}\left(l_{2}\right)\right]\right)$.

Let us call $q_{1}^{\prime}$ the point in $\gamma_{1}$ with $d\left(\gamma_{1}\left(l_{1}\right), q_{1}^{\prime}\right)=d\left(\gamma_{1}\left(l_{1}\right), q_{1}\right)=d\left(\gamma_{1}\left(l_{1}\right), q_{3}\right)=: v_{1}$, and $p_{2}^{\prime}$ the point in $\gamma_{2}$ with $d\left(\gamma_{2}(0), p_{2}^{\prime}\right)=d\left(\gamma_{2}(0), p_{2}\right)=d\left(\gamma_{2}(0), p_{3}\right)=: u_{2}$. We define $u_{1}:=d\left(\gamma_{1}(0), p_{1}\right)=$ $d\left(\gamma_{1}(0), p_{3}\right)$, and $v_{2}:=d\left(\gamma_{2}\left(l_{2}\right), q_{2}\right)=d\left(\gamma_{2}\left(l_{2}\right), q_{3}\right)$. Observe that $d\left(\gamma_{1}(0), \gamma_{2}(0)\right)=u_{1}+u_{2} \leq c$ and $d\left(\gamma_{1}\left(l_{1}\right), \gamma_{2}\left(l_{2}\right)\right)=v_{1}+v_{2} \leq c$.

We can assume that $u_{1}+v_{1} \leq l_{1}=L\left(\gamma_{1}\right)$, since the another case is simpler; this fact implies $u_{2}+v_{2} \leq l_{2}=L\left(\gamma_{2}\right)$. Since $T_{1}$ and $T_{3}$ are $\delta$-fine, we have that $d\left(\gamma_{1}\left(t+u_{1}\right), \gamma_{2}\left(t+u_{2}\right)\right) \leq 2 \delta$, for every $t \in\left[0, l_{1}-u_{1}-v_{1}\right]$. 
Observe that $d\left(\gamma_{1}(t), \gamma_{2}(t)\right) \leq 2 \delta+c$, for every $t \in\left[0, l_{1}-u_{1}-v_{1}\right]$ :

$$
\begin{aligned}
d\left(\gamma_{1}(t), \gamma_{2}(t)\right) & \leq d\left(\gamma_{1}(t), \gamma_{1}\left(t+u_{1}\right)\right)+d\left(\gamma_{1}\left(t+u_{1}\right), \gamma_{2}\left(t+u_{2}\right)\right)+d\left(\gamma_{2}\left(t+u_{2}\right), \gamma_{2}(t)\right) \\
& \leq u_{1}+2 \delta+u_{2} \leq 2 \delta+c
\end{aligned}
$$

If $t \in\left[l_{1}-u_{1}-v_{1}, l_{1}\right]$, we have that

$$
\begin{aligned}
d\left(\gamma_{1}(t), \gamma_{2}(t)\right) \leq & d\left(\gamma_{1}(t), \gamma_{1}\left(l_{1}-u_{1}-v_{1}\right)\right)+d\left(\gamma_{1}\left(l_{1}-u_{1}-v_{1}\right), \gamma_{2}\left(l_{1}-u_{1}-v_{1}\right)\right) \\
& \quad+d\left(\gamma_{2}\left(l_{1}-u_{1}-v_{1}\right), \gamma_{2}(t)\right) \\
\leq & u_{1}+v_{1}+2 \delta+c+u_{1}+v_{1} \leq 2 \delta+5 c
\end{aligned}
$$

Then we have $d\left(\gamma_{1}(t), \gamma_{2}(t)\right) \leq 2 \delta+5 c$, for every $t \in\left[0, l_{1}\right]$.

The same argument with parametrizations which reverse the orientation, gives $d\left(\gamma_{1}(t), \gamma_{2}\left(t+l_{2}-\right.\right.$ $\left.\left.l_{1}\right)\right) \leq 2 \delta+5 c$, for every $t \in\left[0, l_{1}\right]$

Observe now that $t \leq l_{2} t / l_{1} \leq t+l_{2}-l_{1}$, and $l_{2}-l_{1} \leq 2 c$. Consequently we have

$$
d\left(\gamma_{1}(t), \gamma_{2}\left(l_{2} t / l_{1}\right)\right) \leq d\left(\gamma_{1}(t), \gamma_{2}(t)\right)+d\left(\gamma_{2}(t), \gamma_{2}\left(l_{2} t / l_{1}\right)\right) \leq 2 \delta+5 c+l_{2}-l_{1} \leq 2 \delta+7 c
$$

Lemma 13. Each side of $T_{5}$ is a $\left(d_{1}, d_{2}\right)$-quasigeodesic with its canonical parametrization, where $d_{0}:=\left(1+k_{2}\right)\left(1+2 k_{2}\right) k_{4}, d_{1}:=d_{0}\left(1+k_{2}\right)\left(1+2 k_{2}\right)$ and

$$
d_{2}:=\max \left\{k_{1}+\left(1+k_{2}\right) k_{5}, k_{4}\left(15 k_{1}+32 k_{6}\right)+k_{5}, d_{0}^{-1}\left(17 k_{1}+32 k_{6}\right)+2\left(k_{1}+k_{5}\right)+\left(1+2 k_{2}\right)^{-1} k_{5}\right\} .
$$

Proof. Let us consider a side $g_{4}: I \longrightarrow X$ in $T_{4}$ with its arc-length parametrization, and its corresponding side $g_{5}$ in $T_{5}$ with its canonical parametrization.

Given $s, t \in I$, let us choose a geodesic $\gamma$ in $Y$ between $g_{5}(s)$ an $g_{5}(t)$.

By hypothesis, $\gamma$ meets at most a finite number of $\sigma_{n m}^{i}$ 's. Let us assume first that $\gamma$ does not meet $\cup_{n, m, i} \sigma_{n m}^{i}$. Then $\gamma \subseteq Y_{n}$, for some $n \in \Lambda$, and we have by $(c 3)$ and $(c 4)$

$$
d_{Y}\left(g_{5}(t), g_{5}(s)\right)=d_{Y_{n}}\left(g_{5}(t), g_{5}(s)\right) \geq k_{4}^{-1} d_{X_{n}}\left(g_{4}(t), g_{4}(s)\right)-k_{5} \geq k_{4}^{-1} d_{X}\left(g_{4}(t), g_{4}(s)\right)-k_{5}
$$

Let us assume now that $\gamma$ meets $\cup_{n, m, i} \sigma_{n m}^{i}$. Our goal is to split $\gamma$ into some curves joining two closed sets $\sigma_{n m}^{i}$ and $\sigma_{n k}^{j}$ in $Y_{n}$, so that we can relate them with the geodesics $\gamma_{m n k}^{i j} \subseteq X_{n}$ joining $\eta_{n m}^{i}$ with $\eta_{n k}^{j}$ mentioned in $(c 3)$ for $n \in F_{1}$; if $n \in F_{2}$ we can take as $\gamma_{m n k}^{i j}$ any geodesic joining $\eta_{n m}^{i}$ with $\eta_{n k}^{j}$. If $\gamma:[\alpha, \beta] \longrightarrow Y$, let us define

$$
v_{1}^{1}:=\min \left\{\alpha \leq v \leq \beta: \gamma(v) \in \cup_{n, m, i} \sigma_{n m}^{i}\right\}
$$

There exists this minimum since $\gamma$ is a continuous function in a compact interval and $\gamma \cap\left(\cup_{n, m, i} \sigma_{n m}^{i}\right)$ is a compact set: each $\sigma_{n m}^{i}$ is a closed set and $\gamma$ meets at most a finite number of $\sigma_{n m}^{i}$ 's.

Then $\gamma\left(v_{1}^{1}\right) \in \sigma_{n_{1} m_{1}}^{i_{1}}$, for some $n_{1}, m_{1}, i_{1}$, and we define

$$
v_{1}^{2}:=\max \left\{\alpha \leq v \leq \beta: \gamma(v) \in \sigma_{n_{1} m_{1}}^{i_{1}}\right\}
$$

In a similar way, we define recursively

$$
v_{j}^{1}:=\min \left\{v_{j-1}^{2}<v \leq \beta: \gamma(v) \in \cup_{n, m, i} \sigma_{n m}^{i}\right\}
$$


if $\gamma\left(v_{j}^{1}\right) \in \sigma_{n_{j} m_{j}}^{i_{j}}$, for some $n_{j}, m_{j}, i_{j}$, we take

$$
v_{j}^{2}:=\max \left\{v_{j-1}^{2}<v \leq \beta: \gamma(v) \in \sigma_{n_{j} m_{j}}^{i_{j}}\right\}
$$

We can continue this choice for $1 \leq j \leq r$. We have that

$$
d_{Y}\left(g_{5}(t), g_{5}(s)\right)=L_{Y}(\gamma)=\beta-\alpha \geq v_{1}^{1}-\alpha+\sum_{k=2}^{r}\left(v_{k}^{1}-v_{k-1}^{2}\right)+\beta-v_{r}^{2}
$$

Given $\sigma_{n_{k-1} m_{k-1}}^{i_{k-1}}$ and $\sigma_{n_{k} m_{k}}^{i_{k}}$, we have $n_{k-1}=n_{k}, n_{k-1}=m_{k}, m_{k-1}=n_{k}$ or $m_{k-1}=m_{k}$. Since $\sigma_{n m}^{i}=\sigma_{m n}^{i}$, by simplicity in the notation we can assume that $m_{k-1}=n_{k}$ and that the curve $f_{n_{k-1} n_{k} m_{k}}^{i_{k-1} i_{k}} \circ \gamma_{n_{k-1} n_{k} m_{k}}^{i_{k-1} i_{k}}$ joining $\sigma_{n_{k-1} m_{k-1}}^{i_{k-1}}$ and $\sigma_{n_{k} m_{k}}^{i_{k}}$, is contained in $Y_{n_{k}}$. If $\gamma_{n_{k-1} n_{k} m_{k}}^{i_{k-1} i_{k}}:\left[\alpha_{k}, \beta_{k}\right] \longrightarrow$ $X_{n_{k}}(k=2, \ldots, r)$, then $(c 3)$ and $(c 4)$ give that

$$
\begin{aligned}
k_{4}^{-1} d_{X_{n_{k}}} & \left(\gamma_{n_{k-1} n_{k} m_{k}}^{i_{k-1} i_{k}}\left(\beta_{k}\right), \gamma_{n_{k-1} n_{k} m_{k}}^{i_{k-1} i_{k}}\left(\alpha_{k}\right)\right)-b_{n_{k-1} n_{k} m_{k}}^{i_{k-1} i_{k}} \\
& \leq d_{Y_{n_{k}}}\left(f_{n_{k-1} n_{k} m_{k}}^{i_{k-1} i_{k}}\left(\gamma_{n_{k-1} n_{k} m_{k}}^{i_{k-1} i_{k}}\left(\beta_{k}\right)\right), f_{n_{k-1} n_{k} m_{k}}^{i_{k-1} i_{k}}\left(\gamma_{n_{k-1} n_{k} m_{k}}^{i_{k-1} i_{k}}\left(\alpha_{k}\right)\right)\right) .
\end{aligned}
$$

By $(c 2)$,

$$
\begin{aligned}
d_{Y_{n_{k}}} & \left(f_{n_{k-1} n_{k} m_{k}}^{i_{k-1} i_{k}}\left(\gamma_{n_{k-1} n_{k} m_{k}}^{i_{k-1} i_{k}}\left(\beta_{k}\right)\right), f_{n_{k-1} n_{k} m_{k}}^{i_{k-1} i_{k}}\left(\gamma_{n_{k-1} n_{k} m_{k}}^{i_{k-1} i_{k}}\left(\alpha_{k}\right)\right)\right) \\
& \leq \operatorname{diam}_{Y_{n_{k}}}\left(\sigma_{n_{k-1} m_{k-1}}^{i_{k-1}}\right)+d_{Y_{n_{k}}}\left(\sigma_{n_{k-1} m_{k-1}}^{i_{k-1}}, \sigma_{n_{k} m_{k}}^{i_{k}}\right)+\operatorname{diam}_{Y_{n_{k}}}\left(\sigma_{n_{k} m_{k}}^{i_{k}}\right) \\
& \leq\left(1+2 k_{2}\right) d_{Y_{n_{k}}}\left(\sigma_{n_{k-1} m_{k-1}}^{i_{k-1}}, \sigma_{n_{k} m_{k}}^{i_{k}}\right) .
\end{aligned}
$$

Consequently we have

$$
\begin{aligned}
v_{k}^{1}-v_{k-1}^{2} & \geq d_{Y_{n_{k}}}\left(\sigma_{n_{k-1} m_{k-1}}^{i_{k-1}}, \sigma_{n_{k} m_{k}}^{i_{k}}\right) \\
& \geq\left(1+2 k_{2}\right)^{-1} d_{Y_{n_{k}}}\left(f_{n_{k-1} n_{k} m_{k}}^{i_{k-1} i_{k}}\left(\gamma_{n_{k-1} n_{k} m_{k}}^{i_{k-1} i_{k}}\left(\beta_{k}\right)\right), f_{n_{k-1} n_{k} m_{k}}^{i_{k-1} i_{k}}\left(\gamma_{n_{k-1} n_{k} m_{k}}^{i_{k-1} i_{k_{k}}}\left(\alpha_{k}\right)\right)\right) \\
& \geq\left(1+2 k_{2}\right)^{-1}\left(k_{4}^{-1} d_{X_{n_{k}}}\left(\gamma_{n_{k-1} n_{k} m_{k}}^{i_{k-1} i_{k}}\left(\beta_{k}\right), \gamma_{n_{k-1} n_{k} m_{k}}^{i_{k-1} i_{k}}\left(\alpha_{k}\right)\right)-b_{n_{k-1} n_{k}}^{i_{k-1} i_{k}} m_{k}\right) .
\end{aligned}
$$

We have that $\gamma\left(\left[\alpha, v_{1}^{1}\right]\right) \subseteq Y_{n_{1}}$ or $\gamma\left(\left[\alpha, v_{1}^{1}\right]\right) \subseteq Y_{m_{1}}$, and $\gamma\left(\left[v_{r}^{2}, \beta\right]\right) \subseteq Y_{n_{r}}$ or $\gamma\left(\left[v_{r}^{2}, \beta\right]\right) \subseteq Y_{m_{r}}$. By simplicity in the notation, we can assume that $\gamma\left(\left[\alpha, v_{1}^{1}\right]\right) \subseteq Y_{n_{1}}$ and $\gamma\left(\left[v_{r}^{2}, \beta\right]\right) \subseteq Y_{n_{r}}$. Then Remark 6 before Theorem 2 gives

$$
\begin{aligned}
& v_{1}^{1}-\alpha \geq d_{Y_{n_{1}}}\left(g_{5}(s), \sigma_{n_{1} m_{1}}^{i_{1}}\right) \geq k_{4}^{-1} d_{X_{n_{1}}}\left(g_{4}(s), \eta_{n_{1} m_{1}}^{i_{1}}\right)-k_{1}-k_{5}, \\
& \beta-v_{r}^{2} \geq d_{Y_{n_{r}}}\left(g_{5}(t), \sigma_{n_{r} m_{r}}^{i_{r}}\right) \geq k_{4}^{-1} d_{X_{n_{r}}}\left(g_{4}(t), \eta_{n_{r} m_{r}}^{i_{r}}\right)-k_{1}-k_{5} .
\end{aligned}
$$

Consequently we have

$$
\begin{aligned}
d_{Y}( & \left.g_{5}(t), g_{5}(s)\right) \geq v_{1}^{1}-\alpha+\sum_{k=2}^{r}\left(v_{k}^{1}-v_{k-1}^{2}\right)+\beta-v_{r}^{2} \\
\geq & k_{4}^{-1} d_{X_{n_{1}}}\left(g_{4}(s), \eta_{n_{1} m_{1}}^{i_{1}}\right)-k_{1}-k_{5}+k_{4}^{-1} d_{X_{n_{r}}}\left(g_{4}(t), \eta_{n_{r} m_{r}}^{i_{r}}\right)-k_{1}-k_{5} \\
& +\left(1+2 k_{2}\right)^{-1} \sum_{k=2}^{r}\left(k_{4}^{-1} d_{X_{n_{k}}}\left(\gamma_{n_{k-1} n_{k} m_{k}}^{i_{k-1} i_{k}}\left(\beta_{k}\right), \gamma_{n_{k-1} n_{k} m_{k}}^{i_{k-1} i_{k}}\left(\alpha_{k}\right)\right)-b_{n_{k-1} n_{k} m_{k}}^{i_{k-1} i_{k}}\right) \\
\geq & k_{4}^{-1} d_{X_{n_{1}}}\left(g_{4}(s), \eta_{n_{1} m_{1}}^{i_{1}}\right)+k_{4}^{-1} d_{X_{n_{r}}}\left(g_{4}(t), \eta_{n_{r} m_{r}}^{i_{r}}\right)-2\left(k_{1}+k_{5}\right)-\left(1+2 k_{2}\right)^{-1} k_{5} \\
& +\left(1+2 k_{2}\right)^{-1} k_{4}^{-1} \sum_{k=2}^{r} d_{X_{n_{k}}}\left(\gamma_{n_{k-1} n_{k} m_{k}}^{i_{k-1} i_{k}}\left(\beta_{k}\right), \gamma_{n_{k-1} n_{k} m_{k}}^{i_{k-1} i_{k}}\left(\alpha_{k}\right)\right) .
\end{aligned}
$$


Now we want to obtain a continuous curve $\gamma^{\prime}$ in $X$ joining $g_{4}(s)$ with $g_{4}(t)$.

By $(c 2)$ we can choose geodesics $\gamma_{k}$ in $X_{n_{k+1}}(2 \leq k \leq r-1)$ joining $\gamma_{n_{k-1} n_{k} m_{k}}^{i_{k-1} i_{k}}\left(\beta_{k}\right)$ with $\gamma_{n_{k} n_{k+1}}^{i_{k} i_{k+1}} m_{k+1}\left(\alpha_{k+1}\right)$, such that

$$
\begin{aligned}
L_{X_{n_{k+1}}}\left(\gamma_{k}\right) & =d_{X_{n_{k+1}}}\left(\gamma_{n_{k} n_{k+1} m_{k+1}}^{i_{k} i_{k+1}}\left(\alpha_{k+1}\right), \gamma_{n_{k-1} n_{k} m_{k}}^{i_{k-1} i_{k}}\left(\beta_{k}\right)\right) \\
& \leq k_{2} d_{X_{n_{k+1}}}\left(\gamma_{n_{k} n_{k+1} i_{k+1}}^{i_{k} i_{k+1}}\left(\beta_{k+1}\right), \gamma_{n_{k} n_{k+1}}^{i_{k} i_{k+1}}\left(\alpha_{k+1}\right)\right) .
\end{aligned}
$$

By $(b)$ we can choose a geodesic $\gamma_{1}$ in $X_{n_{1}}$ joining $g_{4}(s)$ with $\gamma_{n_{1} n_{2} m_{2}}^{i_{1} i_{2}}\left(\alpha_{2}\right)$, such that

$$
L_{X_{n_{1}}}\left(\gamma_{1}\right)=d_{X_{n_{1}}}\left(g_{4}(s), \gamma_{n_{1} n_{2} m_{2}}^{i_{1} i_{2}}\left(\alpha_{2}\right)\right) \leq d_{X_{n_{1}}}\left(g_{4}(s), \eta_{n_{1} m_{1}}^{i_{1}}\right)+k_{1}
$$

and a geodesic $\gamma_{r}$ in $X_{n_{r}}$ joining $\gamma_{n_{r-1} n_{r} m_{r}}^{i_{r_{-1}} i_{r}}\left(\beta_{r}\right)$ with $g_{4}(t)$, such that $L_{X_{n_{r}}}\left(\gamma_{r}\right) \leq d_{X_{n_{r}}}\left(g_{4}(t), \eta_{n_{r} m_{r}}^{i_{r}}\right)+$ $k_{1}$.

We consider now the continuous curve $\gamma^{\prime}$ in $X$ joining $g_{4}(s)$ with $g_{4}(t)$ obtained by the juxtaposition of the geodesics $\left\{\gamma_{k}\right\}_{k=1}^{r}$ and $\left\{\gamma_{n_{k-1} n_{k} m_{k}}^{i_{k-1} i_{k}}\right\}_{k=2}^{r}$.

On the one hand, these facts give

$$
\begin{aligned}
d_{X}\left(g_{4}(t), g_{4}(s)\right) \leq & L_{X}\left(\gamma^{\prime}\right) \leq d_{X_{n_{1}}}\left(g_{4}(s), \eta_{n_{1} m_{1}}^{i_{1}}\right)+k_{1}+d_{X_{n_{r}}}\left(g_{4}(t), \eta_{n_{r} m_{r}}^{i_{r}}\right)+k_{1} \\
& +\left(1+k_{2}\right) \sum_{k=2}^{r} d_{X_{n_{k}}}\left(\gamma_{n_{k-1} n_{k}}^{i_{k-1} i_{k}}\left(\beta_{k}\right), \gamma_{n_{k-1} n_{k}}^{i_{k-1} i_{k}}\left(\alpha_{k}\right)\right) \\
\leq & 2 k_{1}+d_{0}\left(2\left(k_{1}+k_{5}\right)+\left(1+2 k_{2}\right)^{-1} k_{5}\right)+d_{0}\left(k_{4}^{-1} d_{X_{n_{1}}}\left(g_{4}(s), \eta_{n_{1} m_{1}}^{i_{1}}\right)\right. \\
& +k_{4}^{-1} d_{X_{n_{r}}}\left(g_{4}(t), \eta_{n_{r} m_{r}}^{i_{r}}\right)+\left(1+2 k_{2}\right)^{-1} k_{4}^{-1} \sum_{k=2}^{r} d_{X_{n_{k}}}\left(\gamma_{n_{k-1} n_{k} m_{k}}^{i_{k-1} i_{k}}\left(\beta_{k}\right), \gamma_{n_{k-1} n_{k} m_{k}}^{i_{k-1} i_{k}}\left(\alpha_{k}\right)\right) \\
& \left.-2\left(k_{1}+k_{5}\right)-\left(1+2 k_{2}\right)^{-1} k_{5}\right) \\
\leq & 2 k_{1}+d_{0}\left(2\left(k_{1}+k_{5}\right)+\left(1+2 k_{2}\right)^{-1} k_{5}\right)+d_{0} d_{Y}\left(g_{5}(t), g_{5}(s)\right)
\end{aligned}
$$

(recall that $\left.d_{0}:=\left(1+k_{2}\right)\left(1+2 k_{2}\right) k_{4}\right)$; then we have Corollary 4 , since so far we have not used that $g_{4}(s)$ and $g_{4}(t)$ belong to the same side of $T_{4}$.

On the other hand, Lemma 12 gives

$$
\left(1+k_{2}\right)^{-1}\left(1+2 k_{2}\right)^{-1}|t-s|-15 k_{1}-32 k_{6} \leq d_{X}\left(g_{4}(t), g_{4}(s)\right) .
$$

Consequently we have

$d_{Y}\left(g_{5}(t), g_{5}(s)\right) \geq d_{0}^{-1}\left(1+k_{2}\right)^{-1}\left(1+2 k_{2}\right)^{-1}|t-s|-d_{0}^{-1}\left(17 k_{1}+32 k_{6}\right)-2\left(k_{1}+k_{5}\right)-\left(1+2 k_{2}\right)^{-1} k_{5}$.

In order to see the other inequality, we consider the domain $I$ of $g_{5}$ and $s, t \in I$, with $s<t$.

If $g_{5}([s, t])$ does not intersect with any $\sigma_{n m}^{i}$, then $g_{5}([s, t]) \subseteq h_{m n k}^{i j}$, for some $m, n, k, i, j$. This fact, $(c 3)$ and $(c 4)$ give

$$
d_{Y}\left(g_{5}(t), g_{5}(s)\right) \leq d_{Y_{n}}\left(g_{5}(t), g_{5}(s)\right) \leq k_{4} d_{X_{n}}\left(g_{4}(t), g_{4}(s)\right)+k_{5} .
$$

In other case, we can split the interval $[s, t]$ into a union of intervals $\left[u_{0}, u_{1}\right] \cup\left(u_{1}, u_{2}\right] \cup \cdots \cup\left(u_{l-1}, u_{l}\right]$, with $l \geq 1$, such that $g_{5}\left(\left(u_{r-1}, u_{r}\right]\right) \subseteq h_{m_{r} n_{r} k_{r}}^{i_{r} j_{r}} \subseteq Y_{n_{r}}(1 \leq r \leq l), u_{0}=s$ and $u_{l}=t$. We have that $g_{5}\left(u_{r}\right)$ is an end point of $h_{m_{r} n_{r} k_{r}}^{i_{r} j_{r}}$; we denote by $g_{5}\left(u_{r-1}+\right)$ the other end point of $h_{m_{r} n_{r} k_{r}}^{i_{r} j_{r}}$. 
By $(b)$ and $(c 2)$ we have that $d_{Y_{n_{r+1}}}\left(g_{5}\left(u_{r}+\right), g_{5}\left(u_{r}\right)\right) \leq k_{2} d_{Y_{n_{r+1}}}\left(g_{5}\left(u_{r+1}\right), g_{5}\left(u_{r}+\right)\right)(1 \leq r \leq$ $l-2)$, and $d_{Y_{n_{l}}}\left(g_{5}\left(u_{l-1}+\right), g_{5}\left(u_{l-1}\right)\right) \leq k_{1}$. These facts, $(c 3),(c 4)$ and Lemma 12 give

$$
\begin{aligned}
d_{Y}\left(g_{5}(t), g_{5}(s)\right) \leq & \sum_{r=0}^{l-1} d_{Y_{n_{r+1}}}\left(g_{5}\left(u_{r+1}\right), g_{5}\left(u_{r}+\right)\right)+\sum_{r=1}^{l-2} d_{Y_{n_{r+1}}}\left(g_{5}\left(u_{r}+\right), g_{5}\left(u_{r}\right)\right) \\
& \quad+d_{Y_{n_{l}}}\left(g_{5}\left(u_{l-1}+\right), g_{5}\left(u_{l-1}\right)\right) \\
\leq & k_{1}+\left(1+k_{2}\right) \sum_{r=0}^{l-1} d_{Y_{n_{r+1}}}\left(g_{5}\left(u_{r+1}\right), g_{5}\left(u_{r}+\right)\right) \\
\leq & k_{1}+\left(1+k_{2}\right) \sum_{r=0}^{l-1}\left(k_{4} d_{X_{n_{r+1}}}\left(g_{4}\left(u_{r+1}\right), g_{4}\left(u_{r}+\right)\right)+b_{r}\right) \\
\leq & k_{1}+\left(1+k_{2}\right) k_{5}+\left(1+k_{2}\right) k_{4} \sum_{r=0}^{l-1}\left|u_{r+1}-u_{r}\right| \leq\left(1+k_{2}\right) k_{4}|t-s|+k_{1}+\left(1+k_{2}\right) k_{5}
\end{aligned}
$$

Consequently we have the result.

\section{References.}

[ARY] Alvarez, V., Rodríguez, J.M., Yakubovich, V.A., Subadditivity of p-harmonic "measure" on graphs, Michigan Mathematical Journal 49 (2001), 47-64.

[B] Bonk, M., Quasi-geodesics segments and Gromov hyperbolic spaces, Geometriae Dedicata 62 (1996), 281-298.

[BHK] Bonk, M., Heinonen, J., Koskela, P., Uniformizing Gromov hyperbolic spaces. Astérisque No. 270 (2001).

[CFPR] Cantón, A., Fernández, J. L., Pestana, D., Rodríguez, J. M., On harmonic functions on trees, Potential Analysis 15 (2001), 199-244.

[FR] Fernández, J. L., Rodríguez, J. M., Area growth and Green's function of Riemann surfaces, Arkiv för matematik 30 (1992), 83-92.

[GH] Ghys, E., de la Harpe, P., Sur les Groupes Hyperboliques d'apres Mikhael Gromov. Progress in Mathematics, Volume 83. Birkhäuser. 1990.

[HS] Holopainen, I., Soardi, P. M., p-harmonic functions on graphs and manifolds, Manuscripta Math. 94 (1997), 95-110.

[K1] Kanai, M., Rough isometries and combinatorial approximations of geometries of non-compact Riemannian manifolds, J. Math. Soc. Japan 37 (1985), 391-413.

[K2] Kanai, M., Rough isometries and the parabolicity of Riemannian manifolds, J. Math. Soc. Japan 38 (1986), 227-238.

[P] Paulin, F., On the critical exponent of a discrete group of hyperbolic isometries. Diff. Geom. Appl. 7 (1997), 231-236.

[PRT] Portilla, A., Rodríguez, J. M., Tourís, E., The topology of balls and Gromov hyperbolicity of Riemann surfaces. Preprint.

[RT] Rodríguez, J. M., Tourís, E., Gromov hyperbolicity through decomposition of metric spaces. Preprint. 
[S] Soardi, P. M., Rough isometries and Dirichlet finite harmonic functions on graphs, Proc. Amer. Math. Soc. 119 (1993), 1239-1248.

Ana Portilla, José M. Rodríguez and Eva Tourís

Departamento de Matemáticas Universidad Carlos III de Madrid

Avenida de la Universidad, 30

28911 Leganés, Madrid, SPAIN

e-mails: apferrei@math.uc3m.es jomaro@math.uc3m.es etouris@math.uc3m.es 